\title{
THE HOMOTOPY TYPE OF A POINCARÉ DUALITY COMPLEX AFTER LOOPING
}

\author{
PIOTR BEBEN AND JIE WU
}

\begin{abstract}
We answer a weaker version of the classification problem for the homotopy types of $(n-2)$-connected closed orientable $(2 n-1)$-manifolds. Let $n \geq 6$ be an even integer, and $X$ be a $(n-2)$-connected finite orientable Poincaré $(2 n-1)$-complex such that $H^{n-1}(X ; \mathbb{Q})=0$ and $H^{n-1}\left(X ; \mathbb{Z}_{2}\right)=0$. Then its loop space homotopy type is uniquely determined by the action of higher Bockstein operations on $H^{n-1}\left(X ; \mathbb{Z}_{p}\right)$ for each odd prime $p$. A stronger result is obtained when localized at odd primes.
\end{abstract}

\section{INTRODUCTION}

A connected space $X$ is said to satisfy Poincaré duality with respect to a coefficient ring $R$ if for some fixed nonzero class $e \in H_{n}(X ; R)$ the cap product

$$
e \cap H^{i}(X ; R) \longrightarrow H_{n-i}(X ; R)
$$

pairing is an isomorphism for each $0 \leq i \leq n$. These isomorphisms put additional restraints on the cohomology ring. If we fix $R$ to be a field, then $H^{i}(X ; R)=0$ for $i>n$, and $H^{n-i}(X ; R) \cong H^{i}(X ; R)$ for $0 \leq i \leq n$. In particular, $H^{n}(X ; R) \cong H^{0}(X ; R) \cong R$, and there are nonsingular cup product pairings

$$
H^{i}(X ; R) \otimes H^{n-i}(X ; R) \stackrel{\cup}{\longrightarrow} H^{n}(X ; R) \cong R
$$

so the maps $H^{n-i}(X ; R) \longrightarrow \operatorname{Hom}\left(H^{i}(X ; R), R\right)$ and $H^{i}(X ; R) \longrightarrow H o m\left(H^{n-i}(X ; R), R\right)$ induced by the above pairing are isomorphisms. Every nonzero element $x \in H^{n-i}(X ; R)$ then corresponds to a nonzeo element $y \in H^{i}(X ; R)$ such that the cup product $x y$ is nonzero in $H^{n}(X ; R) \cong R$.

A $C W$-complex $P$ is said to be an orientable Poincaré complex if it satisfies Poincaré duality with respect to all choices of coefficient ring $R$ (see [10] for the non-orientable definition). We say $P$ is finite if it is finite as a $C W$-complex. The dimension of $P$ is the highest degree $n$ in which there is a nonzero element in its $\mathbb{Z}$-cohomology, and we say $P$ is a Poincaré $n$-complex.

Any closed orientable $n$-manifold has the homotopy type of a finite Poincaré $n$-complex. The classification of homotopy types of manifolds is then fittingly phrased in terms of classification of Poincaré complexes. The usual procedure is to first discard the local properties of manifolds, then use homotopy theoretic techniques to classify homotopy types of Poincaré complexes, with local properties finally coming back into the picture when lifting the classification back to manifolds.

Most work to date has involved the classification of low dimensional manifolds (see [10] for a more complete survey). That 1-connected Poincaré 2-complexes and Poincaré 3-complexes have the homotopy type of a 2 -sphere and 3-sphere respectively is an easy consequence of Poincaré duality and the Hurewicz homomorphism. Milnor [12] showed that the $\mathbb{Z}$-cohomology ring classifies 1-connected Poincaré 4-complexes, while Stöcker [15] gave a list of four algebraic invariants that classify the homotopy types of 1-connected orientable Poincaré 5-complexes. Little is known beyond these dimensions. In the highly connected case, $(n-1)$-connected Poincaré $2 n$-complexes have been classified by Whitehead and Wall [16], while Sasao and Takahashi [14] gave a partial solution for $(n-1)$-connected Poincaré $(2 n+2)$-complexes.

AMS 2010 Mathematics subject classification: Primary 55P35; 55P15; 57N65.

Keywords: Poincaré duality complex, loop space, homotopy classification of manifolds. 
The next step in the order of difficulty is to classify $(n-1)$-connected Poincaré $(2 n+1)$-complexes. Though this is still generally an open problem, we can apply two homotopy theoretic simplifications that make this problem solvable. First, we consider the classification problem after our spaces have been localized at some prime $p$. The motivation here is that localized spaces are much simpler from the perspective of homotopy theory, yet they retain much of the homotopy theoretic information of the original space (never-the-less, it is sometimes possible to lift $p$-local results back to the category of integral spaces). Second, we consider the classification problem after looping our spaces. Here one can often use the associative $H$-space structure on loop spaces to decompose them up to homotopy as a weak product of simpler spaces. Spaces that are not homotopy equivalent sometimes have the same loop space homotopy decompositions, so it is reasonable to expect that a loop space homotopy classification will be simpler. Since there is an adjoint isomorphism $[\Sigma X, Y] \stackrel{\cong}{\longrightarrow}[X, \Omega Y]$ of homotopy groups, a loop space homotopy classification is for many practical purposes as good as a homotopy classification of the original spaces.

Let the homology Bockstein operation

$$
\beta_{r}: H_{\star}\left(X ; \mathbb{Z}_{p}\right) \longrightarrow H_{\star-1}\left(X ; \mathbb{Z}_{p}\right)
$$

be the composite $H_{\star}\left(X ; \mathbb{Z}_{p}\right) \stackrel{\delta_{r}}{\longrightarrow} H_{\star-1}\left(X ; Z_{\mathbb{Z}_{p^{r}}}\right) \stackrel{\rho_{r}}{\longrightarrow} H_{\star-1}\left(X ; \mathbb{Z}_{p}\right)$, where $\rho_{r}$ is the connecting map in the homology long exact sequence associated with the short exact sequence

$$
0 \longrightarrow \mathbb{Z}_{p} r \longrightarrow \mathbb{Z}_{p^{r+1}} \longrightarrow \mathbb{Z}_{p} \longrightarrow 0,
$$

and $\rho_{r}$ is induced by the reduction map $\mathbb{Z}_{p} r \longrightarrow \mathbb{Z}_{p}$. Then $\beta_{r}$ where $1 \leq t \leq r$ detects $\mathbb{Z}_{p^{t}}$ summands in the integral homology of $X$. Taking duals one obtains the cohomology Bocksteins

$$
\beta_{r}: H^{*}\left(X ; \mathbb{Z}_{p}\right) \longrightarrow H^{*+1}\left(X ; \mathbb{Z}_{p}\right)
$$

with similar properties.

We show that under a few assumptions, loop space homotopy types are uniquely determined by rational cohomology and the action of the Bocksteins operations $\beta_{r}$.

Theorem 1.1. Fix $m>2$, and let $M$ and $M^{\prime}$ be $(2 m-2)$-connected closed orientable $(4 m-1)$ manifolds (or more generally finite orientable Poincaré complexes). Consider the following conditions:

(1) $\beta_{i}\left(H^{2 m-1}\left(M ; \mathbb{Z}_{p}\right)\right) \cong \beta_{i}\left(H^{2 m-1}\left(M^{\prime} ; \mathbb{Z}_{p}\right)\right)$ for each $i>0$ and some prime $p$;

(2) $H^{2 m-1}(M ; \mathbb{Q}) \cong H^{2 m-1}\left(M^{\prime} ; \mathbb{Q}\right)$;

(3) $\left.\left.H^{2 m-1}\left(M ; \mathbb{Z}_{2}\right)\right)=H^{2 m-1}\left(M^{\prime} ; \mathbb{Z}_{2}\right)\right)=0$;

(4) $H^{2 m-1}(M ; \mathbb{Q})=H^{2 m-1}\left(M^{\prime} ; \mathbb{Q}\right)=0$.

Then:

(i) There is a homotopy equivalence localized at an odd prime $p$

$$
\Omega M_{(p)} \simeq \Omega M_{(p)}^{\prime}
$$

if and only if conditions (1) and (2) hold.

(ii) If conditions (3) and (4) hold, then there is a homotopy equivalence

$$
\Omega M \simeq \Omega M^{\prime}
$$

if and only if condition (1) holds for all odd primes $p$.

The proof is streamlined by assuming condition (4), though with some extra work it can likely be dropped.

\section{MOD- $p$ Poincaré Complexes}

If $X$ is a 1-connected finite type $C W$-complex and $p$ a prime number, recall that the $p$-localization

map $X \stackrel{\ell}{\longrightarrow} X_{(p)}$ induces a ring isomorphism $H^{*}\left(X ; \mathbb{Z}_{p}\right) \stackrel{\cong}{\longrightarrow} H^{*}\left(X_{(p)} ; \mathbb{Z}_{p}\right)$, and $X_{(p)}$ has a $p$-local $C W$-structure (i.e. consisting of $p$-local cells, whose attaching maps are maps of $p$-localized spheres into $p$-local subcomplexes) with $p$-local cells in one-to-one correspondance with generators of the 
$\mathbb{Z}_{p}$-module $H^{*}\left(X_{(p)} ; \mathbb{Z}_{p}\right)$. Because an orientable Poincaré complex $P$ satisfies Poincaré duality on mod- $p$ homology for any prime $p$, then so does its $p$-localization $P_{(p)}$.

Instead of working with the $p$-localization $P_{(p)}$ of a finite orientable Poincaré complex $P$, it is more convenient to use the more general concept of a mod-p Poincaré complex, which is a finite $p$-local $C W$-complex $Q$ that satisfies Poincaré Duality on its mod- $p$ cohomology. The dimension $n$ is the highest degree in which there is a nonzero element in its mod- $p$ cohomology, and one says $Q$ is finite if it has a finite number of $p$-local cells. The $(n-1)$-skeleton of $Q$ in the $p$-local sense will be denoted by $\bar{Q}$. Because the mod- $p$ cohomology generators of a $p$-local space are in one-to-one correspondance with its $p$-local cells, one can see that $Q$ is the cofiber of some map $S_{(p)}^{n-1} \longrightarrow \bar{Q}$.

Fix $n \geq 3$ and $k \geq 1$. We let $\mathcal{T}_{k, n}$ denote the set of classes of homotopy types of mod- $p$ Poincaré $(2 n-1)$-complexes subject to the following conditions: the class $[W]$ is in $\mathcal{T}_{k, n}^{p}$ if and only if

(1) $W$ is $(n-2)$-connected;

(2) $H^{n-1}\left(W ; \mathbb{Z}_{p}\right)$ has rank $k$.

Fix some class $[W] \in \mathcal{T}_{k, n}^{p}$. By mod- $p$ Poincaré duality and the first condition above, the $\mathbb{Z}_{p^{-}}$ submodule $H^{*}\left(\bar{W} ; \mathbb{Z}_{p}\right)$ of $H^{*}\left(W ; \mathbb{Z}_{p}\right)$ is described by an isomorphism

$$
H^{*}\left(\bar{W} ; \mathbb{Z}_{p}\right) \cong \mathbb{Z}_{p}\left\{x_{i}^{*}, y_{i}^{*} \mid 1 \leq i \leq k\right\},
$$

where $\left|x_{i}^{*}\right|=n-1,\left|y_{i}^{*}\right|=n$. We can and will choose the basis for $H^{*}\left(\bar{W} ; \mathbb{Z}_{p}\right)$ to satisfy the following the conditions: there is an integer $k_{1}$, with $0 \leq k_{1} \leq k$, such that whenever $1 \leq i \leq k_{1}$, the action of the Bockstein operations on $H^{*}\left(\bar{W} ; \mathbb{Z}_{p}\right)$ satisfy

$$
\beta_{r_{i}}\left(x_{i}^{*}\right)=y_{i}^{*}
$$

for some choice of integer $r_{i}>0$ (depending on $i$ ), and whenever $k_{1}<i \leq k$ we have

$$
\beta_{r}\left(x_{i}^{*}\right)=0
$$

for each $r>0$.

Next, recall that for any group $G$, a Moore space $M(G, m)$ is unique up to homotopy type. Thus $M\left(G_{1} \oplus G_{2}, m\right) \simeq M\left(G_{1}, m\right) \vee M\left(G_{2}, m\right)$. Therefore there exists a splitting of $\bar{W}$ as a wedge sum of $p$-localized Moore spaces $P^{n}\left(p^{r_{i}}\right)$ for $i \leq k_{1}$ corresponding to the action of $\beta_{r_{i}}$ on $x_{i}^{*}$, and $p$-localized spheres $S^{n-1}$ and $S^{n}$ corresponding to the generators $x_{i}^{*}$ and $y_{j}^{*}$ for $k_{1}<i \leq k$. In other words

$$
\bar{W} \simeq(p) \bigvee_{1 \leq i \leq k_{1}} P^{n}\left(p^{r_{i}}\right) \vee \bigvee_{k_{1}<i \leq k}\left(S^{n-1} \vee S^{n}\right) .
$$

Fixing some generator $z^{*} \in H^{2 n-1}\left(W ; \mathbb{Z}_{p}\right) \cong \mathbb{Z}_{p}$, the cup product structure on $H^{*}\left(W ; \mathbb{Z}_{p}\right)$ is described by a $k \times k \mathbb{Z}_{p}$-matrix representation

$$
A_{z^{*}}=\left(a_{i j}\right)
$$

where $y_{j}^{*} x_{i}^{*}=a_{i j} z^{*}$. Set $k_{2}=k-k_{1}$. We partition $A_{z^{*}}$ into the block form

$$
A_{z^{*}}=\left(\begin{array}{cc}
B_{z^{*}} & D_{z^{*}} \\
C_{z^{*}} & E_{z^{*}}
\end{array}\right)
$$

where $B_{z^{*}}, C_{z^{*}}, D_{z^{*}}, E_{z^{*}}$ are respectively matrices of dimensions $k_{1} \times k_{1}, k_{2} \times k_{1}, k_{1} \times k_{2}, k_{2} \times k_{2}$. Since Poincaré Duality implies the cup product pairing as described above is nonsingular, the matrix $A_{z^{\star}}$ is nonsingular.

Proposition 2.1. Given a class $[W] \in \mathcal{T}_{k, n}^{p}$ and a generator $z^{*} \in H^{2 n-1}\left(W ; \mathbb{Z}_{p}\right)$ where $n \geq 3$, the nonsingular $A_{z^{*}}$ is such that $C_{z^{*}}$ is the zero $k_{1} \times k_{2}$ matrix, and $B_{z^{*}}$ is symmetric when $n$ is even, and skew symmetric when $n$ is odd.

See Section 4 for a proof. Since a $k \times k$ skew symmetric matrix is nonsingular if and only if $k$ even, the following is immediate:

Corollary 2.2. There exist no classes $[W] \in \mathcal{T}_{2 l+1,2 m+1}^{p}$ such that $H^{2 m-1}(W ; \mathbb{Q})=0$ or $H^{2 m}(W ; \mathbb{Q})=$ 0 . 
We therefore focus on those classes $[W] \in \mathcal{T}_{k, n}^{p}$ for $n$ even. The above statements are proved in the next two sections. We see that the homotopy type of $\Omega W$ is completely classified by rational cohomology and the action of the Bocksteins operations as follows:

Theorem 2.3. Let $[W],\left[W^{\prime}\right] \in \mathcal{T}_{k, 2 m}^{p}$ and $m>2$. Then $\Omega W \simeq \Omega W^{\prime}$ if and only if the following are satisfied:

(1) $H^{2 m-1}(W ; \mathbb{Q}) \cong H^{2 m-1}\left(W^{\prime} ; \mathbb{Q}\right)$;

(2) $\beta_{i}\left(H^{2 m-1}\left(W ; \mathbb{Z}_{p}\right)\right) \cong \beta_{i}\left(H^{2 m-1}\left(W^{\prime} ; \mathbb{Z}_{p}\right)\right)$ for each $i>0$.

The subscript $(p)$ is suppressed until the last section of this paper, and we assume that all our spaces are $p$-local, or localized at $p$ where appropriate, for some fixed odd prime $p$. Any reference to a $C W$-structure on a $p$-local space is always taken to be in the $p$-local sense.

\section{Mod- $p$ Loop Space Homology}

Keep $p$ fixed as an odd prime number. Let $\bar{P}$ be a finite type $C W$-complex, $P$ be the cofiber of some map

$$
\alpha: S^{n-1} \longrightarrow \bar{P}
$$

for some fixed integer $n>3$, and

$$
i: \bar{P} \longrightarrow P
$$

denote the inclusion. Let

$$
\alpha^{\prime}: S^{n-2} \longrightarrow \Omega \bar{P}
$$

be the adjoint of $\alpha$. Since $i \circ \alpha^{\prime}$ is null homotopic, the algebra map

$$
(\Omega i)_{\star}: H_{\star}(\Omega \bar{P} ; R) \longrightarrow H_{\star}(\Omega P ; R)
$$

factors through a map

$$
\theta: H_{*}(\Omega \bar{P} ; R) / I \longrightarrow H_{*}(\Omega P ; R)
$$

for any ring $R$, where $I$ is the two-sided ideal generated by the image of of $\alpha_{\star}^{\prime}$ in degree $n-2$.

Determining the conditions under which $\theta$ is a Hopf Algebra isomorphism is called the cell attachment problem. This has a long history, having been studied by Anick [1], Bubenik [3], Félix and Thomas [5], and Halperin, Hess, and Lemaire [11, 7, 8, 9]. Lemaire [1], for one, found that $\theta$ is a Hopf algebra isomorphism whenever the morphism of graded $R$-vector spaces

$$
\operatorname{Tor}_{p}^{\pi}: \operatorname{Tor}_{p}^{H_{*}(\Omega \bar{P} ; R)}(R, R) \longrightarrow \operatorname{Tor}_{p}^{H_{*}(\Omega \bar{P} ; R) / I}(R, R)
$$

induced by the canonical surjection $H_{*}(\Omega X ; R) \stackrel{\pi}{\longrightarrow} H_{*}(\Omega X ; R) / I$ is bijective, and $R$ is a vector space of characteristic $p$.

Our goal in this section is to determine conditions on the cohomology ring $H_{*}\left(\Omega P ; \mathbb{Z}_{p}\right)$ which make $\theta$ a Hopf algebra isomorphism. Many highly connected mod- $p$ Poincaré complexes, including the ones dealt with in this paper, are covered here. The ideal $I$ is computed in Proposition 3.2 and in Theorem 3.5 we use a Leray-Serre spectral sequence approach to arrive at the Hopf algebra isomorphism $\theta$.

Fix any integer $m>1$ such that $n \geq m$. Assume our $C W$-complex $P$ is $(m-1)$-connected $n$-dimensional, with mod- $p$ reduced homology generated by $a_{1}, \ldots, a_{\ell}$ and $z$, where

$$
m=\left|a_{1}\right| \leq\left|a_{2}\right| \leq \cdots \leq\left|a_{\ell}\right|<|z|=n .
$$

Whenever $\left|a_{i}\right|+\left|a_{j}\right|=n$, let the integer $c_{i j}$ be such that $a_{j}^{*} a_{i}^{*}=c_{i j} z^{*}$, where $a_{i}^{*}, a_{j}^{*}, z^{*}$ are the cohomology duals of $a_{i}, a_{j}$ and $z$, and in addition, we make the following assumptions:

(1) $\operatorname{dim} \bar{P}<\operatorname{dim} P$, meaning $\bar{P}$ is the $(n-1)$-skeleton of $P$;

(2) $3(m-1)>n-2$ and $n$ is odd;

(3) $\bar{P} \simeq \Sigma^{2} X$ for some $X$. 
As $\bar{P}$ is homotopy equivalent to a suspension, cup products are trivial on $\bar{H}^{\star}\left(\bar{P} ; \mathbb{Z}_{p}\right) \subseteq \bar{H}^{*}\left(P ; \mathbb{Z}_{p}\right)$, implying the $c_{i j}$ 's describe the cup-product structure for $\bar{H}^{*}\left(P ; \mathbb{Z}_{p}\right)$.

Consider the mod- $p$ homology Serre spectral sequences $\bar{E}$ and $E$ for the path fibrations of $\bar{P}$ and $P$, and the morphism of spectral sequences

$$
\gamma: \bar{E} \longrightarrow E
$$

induced by the inclusion $\bar{P} \stackrel{i}{\longrightarrow} P$. Here we have

$$
\begin{aligned}
& \bar{E}_{*, *}^{2}=H_{*}\left(\bar{P} ; \mathbb{Z}_{p}\right) \otimes H_{*}\left(\Omega \bar{P} ; \mathbb{Z}_{p}\right), \\
& E_{*, *}^{2}=H_{*}\left(P ; \mathbb{Z}_{p}\right) \otimes H_{*}\left(\Omega P ; \mathbb{Z}_{p}\right) .
\end{aligned}
$$

The corresponding mod- $p$ cohomology spectral sequences are labelled by switching subscripts with superscripts as is standard.

Since $\bar{P}$ is homotopy equivalent to a suspension $\Sigma^{2} X$, the basis elements $a_{i}$ of $H_{*}\left(\bar{P} ; \mathbb{Z}_{p}\right)$ and $H_{*}\left(P ; \mathbb{Z}_{p}\right)$ are transgressive. Thus, let

$$
\begin{aligned}
& u_{i}=\tau\left(a_{i}\right) \in H_{\star}\left(\Omega P ; \mathbb{Z}_{p}\right), \\
& \bar{u}_{i}=\tau\left(a_{i}\right) \in H_{*}\left(\Omega \bar{P} ; \mathbb{Z}_{p}\right)
\end{aligned}
$$

be the transgressions of the $a_{i}$ 's. Note that there is a Hopf algebra isomorphism

$$
H_{*}\left(\Omega \bar{P} ; \mathbb{Z}_{p}\right) \cong T\left(\bar{H}_{*}\left(\Sigma X ; \mathbb{Z}_{p}\right)\right) \cong T\left(\bar{u}_{1}, \ldots, \bar{u}_{\ell}\right) .
$$

Since $\Sigma X$ is a suspension, cup products on $\left.\bar{H}_{*}\left(\Sigma X ; \mathbb{Z}_{p}\right)\right)$ are trivial, implying the algebra generators $\bar{u}_{i}$ are primitive. The algebra map $(\Omega i)_{*}$ satisfies $(\Omega i)_{*}\left(\bar{u}_{\ell}\right)=u_{\ell}$. Then on the second page of spectral sequences,

$$
\begin{aligned}
& \gamma^{2}\left(1 \otimes \bar{u}_{i}\right)=1 \otimes u_{i} \\
& \gamma^{2}\left(a_{i} \otimes 1\right)=a_{i} \otimes 1 .
\end{aligned}
$$

Fix $m^{\prime}$ to be the smallest integer such that there is a $c_{i j}$ prime to $p$ for some $i$ and $j$ satisfying $i \leq j$ and $\left|a_{i}\right|=m^{\prime}$. If no such integer exists, set $m^{\prime}=|z|=n$. We now state some properties of the spectral sequences $\bar{E}$ and $E$.

Proposition 3.1. The following hold:

(i) The kernel of the map

$$
(\Omega i)_{\star}: H_{n-2}\left(\Omega \bar{P} ; \mathbb{Z}_{p}\right) \longrightarrow H_{n-2}\left(\Omega P ; \mathbb{Z}_{p}\right)
$$

is generated by $\alpha_{*}^{\prime}\left(\iota_{n-2}\right)$, where $\iota_{n-2} \in H_{*}\left(S^{n-2} ; \mathbb{Z}_{p}\right) \cong \mathbb{Z}_{p}$ is a generator.

When $m^{\prime}=n$, we have $\alpha_{*}^{\prime}\left(\iota_{n-2}\right)=0$.

(ii) $\bar{d}^{r}\left(\bar{E}_{i, j}^{r}\right)=\{0\}$ for $2 \leq r<i$, and $d^{r}\left(E_{i, j}^{r}\right)=\{0\}$ for $2 \leq r<i$ and $i \neq n$.

(iii) $d^{r}(z \otimes 1)=0$ for $r<m^{\prime}$, so $z \otimes 1$ survives to $E_{n, 0}^{m^{\prime}}$. The differential

$$
d^{m^{\prime}}: E_{n, 0}^{m^{\prime}} \longrightarrow E_{n-m^{\prime}, m^{\prime}-1}^{m^{\prime}}
$$

satisfies

$$
d^{m^{\prime}}(z \otimes 1)= \begin{cases}(-1)^{m^{\prime}} \sum_{\left|a_{i}\right|=m^{\prime},\left|a_{j}\right|=n-m^{\prime}} c_{i j}\left(a_{j} \otimes u_{i}\right), & \text { if } m^{\prime}<n \\ 1 \otimes \tau(z) \neq 0, & \text { if } m^{\prime}=n .\end{cases}
$$

(iv) The map

$$
\gamma^{r}: \bar{E}_{i, j}^{r} \longrightarrow E_{i, j}^{r}
$$

is an isomorphism for $2 \leq r \leq i, j<n-2, i \neq n-m^{\prime}$, and $i \neq n$. It is also an isomorphism for $2 \leq r \leq i, i=n-m^{\prime}$ and $j<m^{\prime}-1$. When $i=0$, it is an isomorphism for $j<n-2$ and all $r \geq 2$. 
(v) When $m^{\prime}<n$, the map

$$
\gamma^{r}: \bar{E}_{n-m^{\prime}, m^{\prime}-1}^{r} \longrightarrow E_{n-m^{\prime}, m^{\prime}-1}^{r}
$$

is an isomorphism for $r>n-m^{\prime}$ and $2 \leq r \leq m^{\prime}$, and the projection of the element

$$
\zeta=(-1)^{m^{\prime}} \sum_{\left|a_{i}\right|=m^{\prime},\left|a_{j}\right|=n-m^{\prime}} c_{i j}\left(a_{j} \otimes \bar{u}_{i}\right)
$$

generates its kernel for $\left(m^{\prime}+1\right) \leq r \leq n-m^{\prime}$.

(vi) The projection of $1 \otimes\left(\alpha^{\prime}\right)_{*}\left(\iota_{n-2}\right)$ to $\bar{E}_{0, n-2}^{r}$ generates the kernel of

$$
\gamma^{r}: \bar{E}_{0, n-2}^{r} \longrightarrow E_{0, n-2}^{r}
$$

for $2 \leq r \leq n-m^{\prime}$.

Proof of part (i). Notice there is the following homotopy commmutative diagram

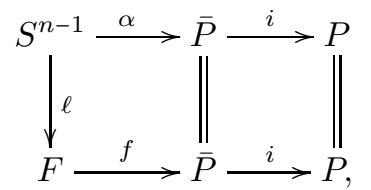

where the top row is the cofibration sequence for the map $\alpha, F$ is the homotopy fiber of the inclusion $\bar{P} \stackrel{i}{\longrightarrow} P$, the bottom row corresponding homotopy fibration sequence, and $\ell$ is some lift. Since $\bar{P} \stackrel{i}{\longrightarrow} P$ induces an isomorphism on mod- $p$ homology in degrees less than $n, F$ is at least $(n-2)$ connected. It is well known that fibers and cofibers agree in the stable range. That is, the lift $\ell$ induces an isomorphism on mod- $p$ homology in degrees less than $m+n-1$. Thus $\ell$ is an inclusion into the bottom sphere inducing in isomorphism in degree $n-1 \bmod -p$ homology, and the adjoint $S^{n-2} \stackrel{\ell^{\prime}}{\longrightarrow} \Omega F$ of $\ell$ induces an isomorphism in degree $n-2$.

By the mod- $p$ homology Serre exact sequence for the homotopy fibration

$$
\Omega F \stackrel{\Omega f}{\longrightarrow} \Omega \bar{P} \stackrel{\Omega i}{\longrightarrow} \Omega P
$$

the image of $(\Omega f)_{*}$ is equal to the kernel of $(\Omega i)_{*}$ in degree $n-2$. By the left homotopy commutative square in diagram (5), $\alpha^{\prime}$ is homotopic to

$$
S^{n-2} \stackrel{\ell^{\prime}}{\longrightarrow} \Omega F \stackrel{\Omega f}{\longrightarrow} \Omega \bar{P} .
$$

Since $\ell^{\prime}$ induces an isomorphism in degree $n-2$, the element $\alpha_{*}^{\prime}\left(\iota_{n-2}\right)$ must generate the kernel of $(\Omega i)_{*}$ in degree $n-2$.

To see that $\alpha_{*}^{\prime}\left(\iota_{n-2}\right)=0$ whenever $m^{\prime}=n$, by part (iv) the map

$$
\gamma^{r}: \bar{E}_{r, n-r-1}^{r} \longrightarrow E_{r, n-r-1}^{r}
$$

is an isomorphism for $r \geq 2$. Since $\bar{E}^{\infty}=E^{\infty}=\{0\}, \bar{E}_{0, n-2}^{r+1}=\frac{\bar{E}_{0, n-2}^{r}}{d^{r}\left(E_{r, n-r-1}^{r}\right)}$, and $E_{0, n-2}^{r+1}=\frac{E_{0, n-2}^{r}}{d^{r}\left(E_{r, n-r-1}^{r}\right)}$, it follows that

$$
\gamma^{r}: \bar{E}_{0, n-2}^{r} \longrightarrow E_{0, n-2}^{r}
$$

is an isomorphism for all $r \geq 2$. Since $\alpha_{*}^{\prime}\left(\iota_{n-2}\right)$ generates the kernel of $(\Omega i)_{*}$ in degree $n-2$, $1 \otimes \alpha_{*}^{\prime}\left(\iota_{n-2}\right) \in \bar{E}_{0, n-2}^{2}$ generates the kernel of $\gamma^{2}$. But $\gamma^{2}$ being an isomorphism implies $1 \otimes \alpha_{*}^{\prime}\left(\iota_{n-2}\right)=0$, so $\alpha_{\star}^{\prime}\left(\iota_{n-2}\right)=0$.

Proof of part (ii). Since path fibrations are principal fibrations, differentials for the spectral sequence of $\bar{E}$ for the path fibration of $\bar{P}$ satisfy

$$
\bar{d}^{r}(a \otimes b)=(1 \otimes b) \bar{d}^{r}(a \otimes 1)
$$

for any $a, b$. Similarly for the spectral sequence $E$ for the path fibration of $P$.

Since the elements $a_{i} \otimes 1 \in \bar{E}_{\left|a_{i}\right|, 0}^{2}$ are transgressive,

$$
\bar{d}^{r}\left(a_{i} \otimes 1\right)=0
$$


for $r<\left|a_{i}\right|$. Since $\gamma^{2}\left(a_{i} \otimes 1\right)=a_{i} \otimes 1 \in E_{\left|a_{i}\right|, 0}^{2}$, the result follows by naturality of spectral sequences.

Proof of part $(i i i)$. In $E_{*, *}^{\left|a_{i}\right|}$ we have the differentials

$$
d_{\left|a_{i}\right|}\left(1 \otimes u_{i}^{*}\right)=a_{i}^{*} \otimes 1, d^{\left|a_{i}\right|}\left(a_{i} \otimes 1\right)=1 \otimes u_{i},
$$

where $u_{i}^{*}$ and $a_{i}^{*}$ are the mod- $p$ cohomology duals. Since we are assuming $\left|a_{1}\right| \leq \cdots \leq\left|a_{\ell}\right|$, we have $\left|a_{1}\right|=\min \left\{\left|a_{1}\right|, \ldots,\left|a_{\ell}\right|\right\}$. When $\left|a_{i}\right|+\left|a_{j}\right|=n$,

$$
\begin{aligned}
d_{m^{\prime}}\left(a_{j}^{*} \otimes u_{i}^{*}\right) & =(-1)^{m^{\prime}}\left(a_{j}^{*} \otimes 1\right) d_{m^{\prime}}\left(1 \otimes u_{i}^{*}\right) \\
& =(-1)^{m^{\prime}}\left(a_{j}^{*} a_{i}^{*}\right) \otimes 1 \\
& =(-1)^{m^{\prime}} c_{i j} z^{*} \otimes 1,
\end{aligned}
$$

and since $c_{i j}$ is divisible by $p$ whenever $\left|a_{j}\right|+\left|a_{i}\right|=n$ and $\left|a_{i}\right|<m^{\prime}$, it follows that $d_{\left|a_{j}\right|}\left(a_{j}^{*} \otimes u_{i}^{*}\right)=0$. Thus, for $r<m^{\prime}$, the differentials

$$
d_{r}: E_{r}^{n-r, r-1} \longrightarrow E_{r}^{n, 0}
$$

in the cohomology spectral sequence for the path fibration of $P$ are zero. By duality of the spectral sequence, $d^{r}(z \otimes 1)=0$ whenever $r<m^{\prime}$, and so we can project $z \otimes 1$ to $E_{n, 0}^{m^{\prime}}$.

When $m^{\prime}=|z|=n$, we see that $z \otimes 1$ is transgressive, with

$$
d^{n}(z \otimes 1)=w
$$

for some nonzero $w \in E_{0, n-1}^{n}$. On the other hand, when $m^{\prime}<n$, we have some integers $c_{i j}^{\prime}$ such that

$$
d^{m^{\prime}}(z \otimes 1)=\sum_{\left|a_{i}\right|=m^{\prime},\left|a_{j}\right|=n-m^{\prime}} c_{i j}^{\prime}\left(a_{j} \otimes u_{i}\right)
$$

From the duality of the spectral sequence,

$$
\begin{aligned}
(-1)^{m^{\prime}} c_{i j}=\left\langle(-1)^{m^{\prime}} c_{i j} z^{*}, z\right\rangle & =\left\langle d_{m^{\prime}}\left(a_{j}^{*} \otimes u_{i}^{*}\right), z\right\rangle \\
& =\left\langle a_{j}^{*} \otimes u_{i}^{*}, d^{m^{\prime}}(z \otimes 1)\right\rangle \\
& =\left\langle a_{j}^{*} \otimes u_{i}^{*}, \sum_{\left|a_{s}\right|=m^{\prime},\left|a_{t}\right|=n-m^{\prime}} c_{s t}^{\prime} a_{s} \otimes u_{t}\right\rangle=c_{i j}^{\prime} .
\end{aligned}
$$

Proof of part $(i v)$. Since the inclusion $\bar{P} \stackrel{i}{\longrightarrow} P$ induces isomorphisms $H_{i}(\bar{P}) \stackrel{i_{*}}{\longrightarrow} H_{i}(P)$ for $i \neq n$, by the Serre homology exact sequences for the path fibrations of $\bar{P}$ and $P$, the map $\Omega \bar{P} \stackrel{\Omega i}{\longrightarrow} \Omega P$ induces isomorphisms $H_{i}(\Omega \bar{P}) \stackrel{(\Omega i)_{*}}{\longrightarrow} H_{i}(\Omega P)$ for $i<n-2$. Therefore $\bar{E}_{i, j}^{2} \stackrel{\gamma^{2}}{\longrightarrow} E_{i, j}^{2}$ is an isomorphism for $i \neq n$ and $j<n-2$.

By parts $(i i)$ and $(i i i)$, elements in $E_{i, j}^{r}$ are in the image of a differential $d^{r}$ only when $i=0$, or when $i=n-m^{\prime}, r=m^{\prime}$, and $j \geq m^{\prime}-1$. By part $(i i)$ differentials $d^{r}$ are zero on $E_{i, *}^{r}$ when $i \neq n$ and $2 \leq r<i$. The above also holds true for the spectral sequence $\bar{E}$ in place of $E$. Therefore $\gamma^{2}$ extends to isomorphisms $\bar{E}_{i, j}^{r} \stackrel{\gamma^{r}}{\longrightarrow} E_{i, j}^{r}$ for $2 \leq r \leq i, j<n-2, i \neq n-m^{\prime}$, and $i \neq n$, or when $2 \leq r \leq i, i=n-m^{\prime}$ and $j<m^{\prime}-1$.

For the case $i=0$, suppose by induction $\bar{E}_{0, j}^{r} \stackrel{\gamma^{r}}{\longrightarrow} E_{0, j}^{r}$ is an isomorphism for some $r \geq 2$ and $j<n-2$. This is true when $r=2$. As we saw above $\bar{E}_{r, j-r+1}^{r} \stackrel{\gamma^{r}}{\longrightarrow} E_{r, j-r+1}^{r}$ is an isomorphism when $r \neq n-m^{\prime}, r \neq n$, and $r \geq 2$. Therefore it is an isomorphism for all choices of $(r, j-r+1)$ lying on the positive quadrant with $j<n-2$ (since $j<n-2$ implies $j-r+1<m^{\prime}-1$ whenever $r=n-m^{\prime}$, and $r<n$ whenever $j-r+1=0)$. Since $\bar{E}_{0, j}^{r+1}=\frac{\bar{E}_{0, j}^{r}}{d^{r}\left(\bar{E}_{r, j-r+1}^{r}\right)}$, and $E_{0, j}^{r+1}=\frac{E_{0, j}^{r}}{d^{r}\left(E_{r, j-r+1}^{r}\right)}$, it follows that $\bar{E}_{0, j}^{r+1} \stackrel{\gamma^{r+1}}{\longrightarrow} E_{0, j}^{r+1}$ is also an isomorphism, and the induction is finished. 
Proof of part $(v)$. From the proof of part $(i v), \bar{E}_{n-m^{\prime}, m^{\prime}-1}^{2} \stackrel{\gamma^{2}}{\longrightarrow} E_{n-m^{\prime}, m^{\prime}-1}^{2}$ is an isomorphism. That $\gamma^{2}$ extends to isomorphisms $\bar{E}_{n-m^{\prime}, m^{\prime}-1}^{r} \stackrel{\gamma^{r}}{\longrightarrow} E_{n-m^{\prime}, m^{\prime}-1}^{r}$ for $2 \leq r \leq m^{\prime}$ follows by parts (ii) and (iii). Since $E_{n-m^{\prime}, m^{\prime}-1}^{m^{\prime}+1}=\frac{E_{n-m^{\prime}, m^{\prime}-1}^{m^{\prime}}}{d^{m^{\prime}}(z \otimes 1)}, \zeta$ generates the kernel of $\bar{E}_{n-m^{\prime}, m^{\prime}-1}^{m^{\prime}+1} \stackrel{\gamma^{m^{\prime}}}{\longrightarrow} E_{n-m^{\prime}, m^{\prime}-1}^{m^{\prime}+1}$.

We have $\bar{E}_{n-m^{\prime}, m^{\prime}-1}^{r} \cong \bar{E}_{n-m^{\prime}, m^{\prime}-1}^{r}$ and $E_{n-m^{\prime}, m^{\prime}-1}^{r} \cong E_{n-m^{\prime}, m^{\prime}-1}^{r}$ for $m^{\prime}+1 \leq r \leq n-m^{\prime}$ following from part (ii). Hence the projection of $\zeta$ generates the kernel of $\bar{E}_{n-m^{\prime}, m^{\prime}-1}^{r} \stackrel{\gamma^{r}}{\longrightarrow} E_{n-m^{\prime}, m^{\prime}-1}^{r}$ for $m^{\prime}+1 \leq r \leq n-m^{\prime}$.

Proof of part $(v i)$. By part $(i),\left(\alpha^{\prime}\right)_{*}\left(\iota_{n-2}\right)$ generates the kernel of $(\Omega i)_{*}$, so $1 \otimes\left(\alpha^{\prime}\right)_{*}\left(\iota_{n-2}\right)$ generates the kernel of $\bar{E}_{0, n-2}^{2} \stackrel{\gamma^{2}}{\longrightarrow} E_{0, n-2}^{2}$.

Fix some $2 \leq r<n-m^{\prime}$, and suppose the projection of $1 \otimes\left(\alpha^{\prime}\right)_{*}\left(\iota_{n-2}\right)$ generates the kernel of $\bar{E}_{0, n-2}^{r} \stackrel{\gamma^{r+1}}{\longrightarrow} E_{0, n-2}^{r}$.

Recall from part (iv) that $\bar{E}_{r, n-r-1}^{r} \stackrel{\gamma^{r}}{\longrightarrow} E_{r, n-r-1}^{r}$ is an isomorphism for $2 \leq r<n-m^{\prime}$. Then because $\bar{E}_{0, n-2}^{r+1}=\frac{\bar{E}_{0, n-2}^{r}}{d^{r}\left(\bar{E}_{r, n-r-1}^{r}\right)}$ and $E_{0, n-2}^{r+1}=\frac{E_{0, n-2}^{r}}{d^{r}\left(E_{r, n-r-1}^{r}\right)}$, the projection of $1 \otimes\left(\alpha^{\prime}\right)_{*}\left(\iota_{n-2}\right)$ also generates the kernel of $\bar{E}_{0, n-2}^{r+1} \stackrel{\gamma^{r+1}}{\longrightarrow} E_{0, n-2}^{r+1}$, and the result follows by induction.

For elements $x$ and $y$ in a graded algebra, let $[x, y]=x y-(-1)^{|x| y \mid} y x$ denote the graded Lie bracket. In the next lemma we use the following sets for indexing:

$$
\begin{gathered}
\mathcal{A}_{s, k}=\left\{(i, j)|k<i<j \leq \ell,| a_{i}|=s,| a_{j} \mid=n-s\right\}, \\
\mathcal{B}_{k}=\bigcup_{s} \mathcal{A}_{s, k}=\left\{(i, j)|k<i<j \leq \ell,| a_{i}|+| a_{j} \mid=n\right\} .
\end{gathered}
$$

Proposition 3.2. Set $\eta=\left\lceil\frac{n}{2}\right\rceil$. Consider the following elements in $H_{n-2}\left(\Omega \bar{P} ; \mathbb{Z}_{p}\right)$ for $m \leq s \leq \eta$ :

$$
\kappa_{s}=\sum_{(i, j) \in \mathcal{A}_{s, 0}} c_{i j}\left[\bar{u}_{i}, \bar{u}_{j}\right]
$$

Let $m^{\prime} \geq m$ be the smallest integer such that there is a $c_{i j}$ prime to $p$ for some $i \leq j$, with $i$ satisfying $\left|a_{i}\right|=m^{\prime}$. If no such integer exists, set $m^{\prime}=|z|=n$.

Then there exist integers $b_{m}, b_{m+1}, \ldots, b_{\eta}$, each prime to $p$, such that

$$
\alpha_{\star}^{\prime}\left(\iota_{n-2}\right)=\sum_{s=m}^{\eta}(-1)^{s} b_{s} \kappa_{s} .
$$

We will prove Proposition 3.2 using an inductive argument on the skeleta of $P$. We describe the setup for this before going into the proof.

Since $\left|a_{1}\right| \leq \cdots \leq\left|a_{\ell}\right|$, one can take the subcomplex $Y_{k}$ of $\bar{P}$, with $H_{*}\left(Y_{k} ; \mathbb{Z}_{p}\right)$ generated by $a_{1}, \ldots, a_{k}$. Here $Y_{\ell}=\bar{P}$, and $Y_{0}$ is a point. Let us consider the quotients $P / Y_{k}$ and $\bar{P} / Y_{k}$. Note $P / Y_{0}=P$, $\bar{P} / Y_{0}=\bar{P}, P / Y_{\ell}=S^{n}$, and $\bar{P} / Y_{\ell}=*$. Abusing notation, $P / Y_{k}$ has reduced mod- $p$ homology generated by $a_{k+1}, \ldots, a_{\ell}$, and the single degree $n$ generator $z$. The non-trivial cup products on $\bar{H}^{*}\left(P / Y_{k} ; \mathbb{Z}_{p}\right)$ are described by $a_{j}^{*} a_{i}^{*}=c_{i j} z^{*}$ whenever $\left|a_{j}\right|+\left|a_{i}\right|=n$, and $j \geq k$. We fix $m_{k}$ to be the smallest integer so that there is a $c_{i j}$ prime to $p$ for some $i$ and $j$ satisfying $k \leq i \leq j$ and $\left|a_{i}\right|=m_{k}$. If no such integer exists, set $m_{k}=|z|=n$.

The $(n-1)$-skeleton $P / Y_{k}$ is $\bar{P} / Y_{k}$. Let $\alpha_{k}: S^{n-1} \longrightarrow \bar{P} / Y_{k}$ be the attaching map for the single $n$-cell of $P / Y_{k}$,

$$
\alpha_{k}^{\prime}: S^{n-2} \longrightarrow \Omega \bar{P} / Y_{k}
$$

be the adjoint of $\alpha_{k}$, and

$$
i_{k}: \bar{P} / Y_{k} \longrightarrow P / Y_{k}
$$

the inclusion of the $(n-1)$-skeleton. 
Let $\left(\bar{E}_{k}\right)$ and $\left(E_{k}\right)$ be the mod- $p$ homology spectral sequence for the path-space fibration of $\bar{P} / Y_{k}$ and $P / Y_{k}$ respectively, and

$$
\gamma:\left(\bar{E}_{k}\right) \longrightarrow\left(E_{k}\right)
$$

be the morphism of spectral sequences induced by $i_{k}$.

Since $\bar{P}$ is a double suspension, so is $\bar{P} / Y_{k}$. Just as before, we have generators $u_{k+1}, \ldots, u_{\ell} \epsilon$ $H_{*}\left(\Omega P / Y_{k-1} ; \mathbb{Z}_{p}\right)$, and $\bar{u}_{k+1}, \ldots, \bar{u}_{\ell} \in H_{*}\left(\Omega\left(\bar{P} / Y_{k-1}\right) ; \mathbb{Z}_{p}\right)$, that are the trangressives of $a_{k+1}, \ldots, a_{\ell}$, with the $\bar{u}_{i}$ 's being primitive.

Remark 3.3. The spaces $P / Y_{k}$ satisfy the same basic properties as $P$ outlined at the beginning of the section. Then Proposition 3.1 applies for $P / Y_{k}$ in place of $P$.

More precisely, in Proposition 3.1 we can take $P / Y_{k}, \bar{P} / Y_{k}, \alpha_{k}, i_{k},\left(\bar{E}_{k}\right),\left(E_{k}\right)$, and $m_{k}$ in place of $P, \bar{P}, \alpha, i, \bar{E}, E$, and $m^{\prime}$ respectively. The sums in parts (ii) and (iii) of Proposition 3.1 are taken with respect to the basis elements $a_{k+1}, \ldots, a_{\ell}$ of $H_{*}\left(P / Y_{k} ; \mathbb{Z}_{p}\right)$.

Proof of Proposition 3.2. The proof proceeds using induction. At each stage we show that the proposition holds for each quotient $P / Y_{k}$ in place of $P$. The induction starts with the base case $P / Y_{\ell}=S^{n}$ and ends with $P / Y_{0}=P$.

Assume Proposition 3.2 holds for the quotient $P / Y_{k}$, for some $1 \leq k \leq \ell$. That is, let us assume $\left(\alpha_{k}^{\prime}\right)_{*}\left(\iota_{n-2}\right)=\chi_{k}$, where we set

$$
\chi_{k}=\sum_{s=\left|a_{k+1}\right|}^{\eta}(-1)^{s} b_{s} \kappa_{s, k}
$$

and

$$
\kappa_{s, k}=\sum_{(i, j) \in \mathcal{A}_{s, k}} c_{i j}\left[\bar{u}_{i}, \bar{u}_{j}\right]
$$

in $H_{*}\left(\Omega\left(\bar{P} / Y_{k}\right) ; \mathbb{Z}_{p}\right)$. The base case $k=\ell$ and $P / Y_{\ell}=S^{n}$ is clearly true.

Since $\bar{P} / Y_{k-1}$ is a double suspension, the elements $\bar{u}_{k}, \ldots, \bar{u}_{\ell}$ in

$$
H_{*}\left(\Omega\left(\bar{P} / Y_{k-1}\right) ; \mathbb{Z}_{p}\right) \cong T\left(\bar{u}_{k}, \ldots, \bar{u}_{\ell}\right)
$$

are primitive. Since $3(m-1)>n-2, H_{n-2}\left(\Omega\left(\bar{P} / Y_{k-1}\right) ; \mathbb{Z}_{p}\right)$ has no monomials of length greater than 2 , and so the brackets $\left[\bar{u}_{i}, \bar{u}_{j}\right]$ subject to $(i, j) \in \mathcal{B}_{k-1}$ form a basis for the primitives in $H_{n-2}\left(\Omega\left(\bar{P} / Y_{k}\right) ; \mathbb{Z}_{p}\right)$ (note that $n$ is odd implies $\left.i \neq j\right)$. Because $\iota_{n-2}$ is primitive, $\left(\alpha_{k-1}^{\prime}\right)_{*}\left(\iota_{n-2}\right)$ is a primitive element in $H_{n-2}\left(\Omega\left(\bar{P} / Y_{k-1}\right) ; \mathbb{Z}_{p}\right)$, and so for some integers $c_{i j}^{\prime \prime}$ we can set

$$
\left(\alpha_{k-1}^{\prime}\right)_{*}\left(\iota_{n-2}\right)=\sum_{(i, j) \in \mathcal{B}_{k-1}} c_{i j}^{\prime \prime}\left[\bar{u}_{i}, \bar{u}_{j}\right] .
$$

Take the quotient map

$$
q_{k-1}: \bar{P} / Y_{k-1} \longrightarrow \bar{P} / Y_{k} .
$$

Notice that $\alpha_{k}$ factors as $q_{k-1} \circ \alpha_{k-1}$, so $\alpha_{k}^{\prime}$ factors as

$$
\alpha_{k}^{\prime}: S^{n-2} \stackrel{\alpha_{k-1}^{\prime}}{\longrightarrow} \Omega\left(\bar{P} / Y_{k-1}\right) \stackrel{\Omega q_{k-1}}{\longrightarrow} \Omega\left(\bar{P} / Y_{k}\right) .
$$

Since the algebra map $\left(\Omega q_{k-1}\right)_{*}$ sends $\bar{u}_{i}$ to $\bar{u}_{i}$ for $i>k$, and $\bar{u}_{k}$ to 0 , in $H_{n-2}\left(\Omega\left(\bar{P} / Y_{k}\right) ; \mathbb{Z}_{p}\right)$ we have

$$
\left(\alpha_{k}^{\prime}\right)_{*}\left(\iota_{n-2}\right)=\sum_{(i, j) \in \mathcal{B}_{k}} c_{i j}^{\prime \prime}\left[\bar{u}_{i}, \bar{u}_{j}\right] .
$$

But $\left(\alpha_{k}^{\prime}\right)_{*}\left(\iota_{n-2}\right)=\chi_{k}$ by our inductive assumption, so by comparing coefficients

$$
c_{i j}^{\prime \prime}=(-1)^{\left|a_{i}\right|} b_{\left|a_{i}\right|} c_{i j}
$$

whenever $(i, j) \in \mathcal{B}_{k}$. That is, whenever $k<i<j \leq \ell$ and $\left|a_{i}\right|+\left|a_{j}\right|=n$. Therefore, in order to show

$$
\left(\alpha_{k-1}^{\prime}\right)_{*}\left(\iota_{n-2}\right)=\chi_{k-1}=\sum_{s=\left|a_{k}\right|}^{\eta}(-1)^{s} b_{s} \kappa_{s, k-1},
$$

where

$$
\kappa_{s, k-1}=\sum_{(i, j) \in \mathcal{A}_{s, k-1}} c_{i j}\left[\bar{u}_{i}, \bar{u}_{j}\right],
$$


we note that $\mathcal{A}_{\left|a_{k}\right|, k} \subseteq \mathcal{A}_{\left|a_{k}\right|, k-1}$ and $\mathcal{A}_{s, k}=\mathcal{A}_{s, k-1}$ when $s>\left|a_{k}\right|$, and so we are left to show there exists an integer $b_{\left|a_{k}\right|}$ prime to $p$ such that $c_{i j}^{\prime \prime}=(-1)^{\left|a_{k}\right|} b_{\left|a_{k}\right|} c_{i j}$, for $i$ and $j$ satisfying $k \leq i<j$, $\left|a_{i}\right|=\left|a_{k}\right|$, and $\left|a_{i}\right|+\left|a_{j}\right|=n$.

Using Remark 3.3 and part (iii) of Proposition 3.1.

$$
d^{m_{k}}(z \otimes 1)= \begin{cases}(-1)^{m_{k}} \sum_{\left|a_{i}\right|=m_{k},\left|a_{j}\right|=n-m_{k}} c_{i j}\left(a_{j} \otimes u_{i}\right), & \text { if } m_{k}<n \\ 1 \otimes \tau(z), & \text { if } m_{k}=|z|=n\end{cases}
$$

for some nonzero $1 \otimes \tau(z) \in\left(E_{k-1}\right)_{0, n-1}^{m_{k}}$.

By part $(v)$ of Proposition 3.1, the map

$$
\gamma^{r}:\left(\bar{E}_{k-1}\right)_{n-m_{k}, m_{k}-1}^{r} \longrightarrow\left(E_{k-1}\right)_{n-m_{k}, m_{k}-1}^{r}
$$

is an isomorphism for $2 \leq r \leq m_{k}$, and the following element

$$
\zeta=(-1)^{m_{k}} \sum_{\left|a_{i}\right|=m_{k},\left|a_{j}\right|=n-m_{k}} c_{i j}\left(a_{j} \otimes \bar{u}_{i}\right)
$$

in $\left(\bar{E}_{k-1}\right)_{n-m_{k}, m_{k}-1}^{r}$, with the sum taken with respect to the basis $a_{k}, \ldots, a_{\ell}$ of $H_{*}\left(\bar{P} / Y_{k-1} ; \mathbb{Z}_{p}\right)$, generates the kernel of $\gamma^{r}$ for $m_{k}+1 \leq r \leq n-m_{k}$ (note: $\bar{d}^{r}\left(\bar{E}_{k-1}\right)_{n-m_{k}, m_{k}-1}^{r}=\{0\}$ for $r$ in this range, so we can project $\zeta$ ).

By part $(i)$ of Proposition 3.1. $\left(\alpha_{k-1}^{\prime}\right)_{*}\left(\iota_{n-2}\right)$ generates the kernel of

$$
\left(\Omega i_{k-1}\right)_{*}: H_{n-2}\left(\Omega\left(\bar{P} / Y_{k-1}\right) ; \mathbb{Z}_{p}\right) \stackrel{\Omega i_{k-1}}{\longrightarrow} H_{n-2}\left(\Omega\left(P / Y_{k-1}\right) ; \mathbb{Z}_{p}\right),
$$

and by part $(v i)$, the projection of $1 \otimes\left(\alpha_{k-1}^{\prime}\right)_{*}\left(\iota_{n-2}\right)$ generates the kernel of

$$
\gamma^{r}:\left(\bar{E}_{k-1}\right)_{0, n-2}^{r} \longrightarrow\left(E_{k-1}\right)_{0, n-2}^{r}
$$

for $2 \leq r \leq n-m_{k}$. In particular

$$
\gamma^{r}\left(1 \otimes\left(\alpha_{k-1}^{\prime}\right)_{*}\left(\iota_{n-2}\right)\right)=0
$$

for $r \geq 2$.

We now return to showing there exists a $b_{\left|a_{k}\right|}$ prime to $p$ such that $c_{k j}^{\prime \prime}=(-1)^{\left|a_{k}\right|} b_{\left|a_{k}\right|} c_{i j}$ for $i$ and $j$ such that $k \leq i<j,\left|a_{i}\right|=\left|a_{k}\right|$, and $\left|a_{i}\right|+\left|a_{j}\right|=n$. The case $m_{k}=|z|=n$ is easy. Here we have $c_{i j}=0$ for each choice of $i, j$, and $\left(\alpha_{k-1}^{\prime}\right)_{*}\left(\iota_{n-2}\right)=0$ by Remark 3.3 and part $(i)$ of Proposition 3.1. Then $c_{k j}^{\prime \prime}=0$, and we can set $b_{\left|a_{k}\right|}=1$. Let us therefore focus on the case $m_{k}<n$. Fix $q=\left|a_{k}\right|$. By definition $m_{k} \geq q$, and since $m_{k}<n$ and $n$ is odd, $q<n-q$.

Let us again recall that path fibrations are principal fibrations, and so our differentials satisfy $d^{r}(a \otimes b)=(1 \otimes b) d^{r}(a \otimes 1)$ and $\bar{d}^{r}(a \otimes b)=(1 \otimes b) \bar{d}^{r}(a \otimes 1)$.

Consider the following element

$$
\zeta^{\prime \prime}=\sum_{\left|a_{i}\right|=q,\left|a_{j}\right|=n-q} c_{i j}^{\prime \prime}\left(a_{j} \otimes \bar{u}_{i}\right)
$$

in $\left(\bar{E}_{k-1}\right)_{n-q, q-1}^{r}$ for $2 \leq r \leq n-q$. Since $d^{\left|a_{i}\right|}\left(a_{i} \otimes \bar{u}_{j}\right)=1 \otimes \bar{u}_{j} \bar{u}_{i}$ in $\left(\bar{E}_{k-1}\right)_{0, n-2}^{\left|a_{i}\right|}$, then $1 \otimes \bar{u}_{j} \bar{u}_{i}=0$ in $\left(\bar{E}_{k-1}\right)_{0, n-2}^{r}$ when $r>\left|a_{i}\right|$, and so $1 \otimes\left[\bar{u}_{i}, \bar{u}_{j}\right]=1 \otimes \bar{u}_{i} \bar{u}_{j}$ in $\left(\bar{E}_{k-1}\right)_{0, n-2}^{r}$ under the condition that $\left|a_{i}\right|<\left|a_{j}\right|$. Because $q=\left|a_{k}\right|=\min \left\{a_{k}, \ldots, a_{\ell}\right\}, n-q$ is the largest possible degree of an element $a_{j} \in\left\{a_{k+1}, \ldots, a_{\ell}\right\}$ such that $\left|a_{i}\right|+\left|a_{j}\right|=n$ for some other element $a_{i}$. Then $1 \otimes\left(\alpha_{k-1}^{\prime}\right)_{*}\left(\iota_{n-2}\right)=0$ in $\left(\bar{E}_{k-1}\right)_{0, n-2}^{r}$ for $r>n-q$ since it cannot be in the image of any differential. Therefore in $\left(\bar{E}_{k-1}\right)_{0, n-2}^{n-q}$

$$
\begin{aligned}
1 \otimes\left(\alpha_{k-1}^{\prime}\right)_{*}\left(\iota_{n-2}\right) & =\sum_{\substack{\left|a_{i}\right|=q,\left|a_{j}\right|=n-q \\
d^{n-q}\left(\zeta^{\prime \prime}\right) .}} c_{i j}^{\prime \prime}\left(1 \otimes \bar{u}_{i} \bar{u}_{j}\right) \\
& ={ }^{n-q}
\end{aligned}
$$

Since $q<n-q$, no nonzero element in $\left(E_{k-1}\right)_{n-q, q-1}^{n-q}$ is in the image of the differential $d^{n-q}$. Likewise, no nonzero element $\left(\bar{E}_{k-1}\right)_{n-q, q-1}^{n-q}$ is in the image of the differential $\bar{d}^{n-q}$. Since $\left(E_{k-1}\right)_{n-q, q-1}^{\infty}=$ $\{0\}$ and $\left(\bar{E}_{k-1}\right)_{n-q, q-1}^{\infty}=\{0\}$, the differentials

$$
\begin{aligned}
& \bar{d}^{n-q}:\left(\bar{E}_{k-1}\right)_{n-q, q-1}^{n-q} \longrightarrow\left(\bar{E}_{k-1}\right)_{0, n-2}^{n-q} \\
& d^{n-q}:\left(E_{k-1}\right)_{n-q, q-1}^{n-q} \longrightarrow\left(E_{k-1}\right)_{0, n-2}^{n-q}
\end{aligned}
$$


must both be injections. Now because $\bar{d}^{n-q}\left(\zeta^{\prime \prime}\right)=1 \otimes\left(\alpha_{k-1}^{\prime}\right)_{*}\left(\iota_{n-2}\right)$ in $\left(\bar{E}_{k-1}\right)_{0, n-2}^{n-q}$, and $\gamma^{n-q}(1 \otimes$ $\left.\left(\alpha_{k-1}^{\prime}\right)_{*}\left(\iota_{n-2}\right)\right)=0$, then

$$
\gamma^{n-q}\left(\zeta^{\prime \prime}\right)=\sum_{\left|a_{i}\right|=q,\left|a_{j}\right|=n-q} c_{i j}^{\prime \prime}\left(a_{j} \otimes u_{i}\right)=0 .
$$

Now suppose $q=m_{k}$, where again we recall $q=\left|a_{k}\right|$. In this case the projection of $1 \otimes\left(\alpha_{k-1}^{\prime}\right)_{*}\left(\iota_{n-2}\right)$ generates the kernel of $\gamma^{r}$ as in equation (10). Then by naturality of the spectral sequences $\zeta^{\prime \prime}$ generates the kernel of

$$
\gamma^{n-q}=\gamma^{n-m_{k}}: \bar{E}_{n-m_{k}, m_{k}-1}^{n-m_{k}} \longrightarrow E_{n-m_{k}, m_{k}-1}^{n-m_{k}} .
$$

But as we saw before, the kernel of this is also generated by $\zeta$, so we must have $\zeta^{\prime \prime}=b \zeta$ for some integer $b$ prime to $p$. Comparing coefficients in equations (9) and (11), we set $b_{\left|a_{k}\right|}=b$, and we have $c_{i j}^{\prime \prime}=b_{\left|a_{k}\right|}(-1)^{m_{k}} c_{i j}$ for $i$ and $j$ such that $k \leq i<j,\left|a_{i}\right|=m_{k}$, and $\left|a_{j}\right|=n-m_{k}$. Therefore equation (6) holds in this case.

Next suppose $q<m_{k}$. By part (iv) of Proposition 3.1

$$
\gamma^{n-q}: \bar{E}_{n-q, q-1}^{n-q} \longrightarrow E_{n-q, q-1}^{n-q}
$$

is an isomorphism. Since $\gamma^{n-q}\left(\zeta^{\prime \prime}\right)=0$, we must have $c_{i j}^{\prime \prime}=0$ for each of the coefficients of $\zeta^{\prime \prime}$. Then we can choose $b_{\left|a_{k}\right|}=1$ for example, and the result follows as the previous case. This finishes the induction.

Lemma 3.4. Let $V=F\left\{x_{1}, \ldots, x_{k}\right\}$ be a vector space over a field $F$, and $T(V)$ the tensor algebra generated by $V$. For an odd integer $N$, consider a nonzero homogeneous element in $T(V)$

$$
\xi=\sum_{\left|x_{i}\right|+\left|x_{j}\right|=N} b_{i j} x_{i} x_{j}
$$

satisfying the condition $b_{i j} \neq 0$ if and only if $b_{j i} \neq 0$, and let $I$ be the two-sided ideal generated by $\xi$.

Take a nonzero homogeneous element in $T(V)$

$$
u=\sum_{\left|x_{i}\right|=M} a_{i} x_{i}
$$

for some integer $M$ and integers $a_{i}$. Then for any element $w \in T(V), w u \in I$ if and only if $w \in I$.

Proof. We will say an element $w \in T(V)$ has length $l$ if it is a linear combination of monomials in $T(V)$ of length at most $l$. This gives a filtration of $T(V)$ by length.

Since $w \in I$ implies $w u \in I$, it remains to show that $w \notin I$ implies $w u \notin I$. The proof is by induction on length of elements in $T(V)$. Assume $w \notin I$ implies $w u \notin I$ for all $w \in T(V)$ of length $l$. The base case $l=0$ is clearly true since $u$ is not in $I$. The case $l=1$ is also obviously true since $\xi$ must have summands $b_{i j} x_{i} x_{j}$ with $b_{i j} \neq 0$ and $\left|x_{j}\right| \neq M$, which $w u$ cannot have (this follows from the fact that $\xi \neq 0$, that $N$ is odd, and the property $b_{i j} \neq 0$ if and only if $\left.b_{j i} \neq 0\right)$.

Consider a nonzero $w \in T(V)$ of length $l+1$ such that $w \notin I$. Let us assume $w u \in I$. Using the inductive assumption we will show this leads to a contradiction.

Note $w u$ has length $l+2$ and and $\xi$ has length 2 . Since $w u \in I$, we can write

$$
w u=\sum_{j} v_{j} x_{j}+v \xi
$$

with $v$ of length $l$, and each $v_{j}$ is some (possibly zero) element in $I$ length $l+1$.

Observe $v \neq 0$, for otherwise $v \xi=0$, and so we would have $v_{j}=0$ whenever $\left|x_{j}\right| \neq M$, and $v_{j}=a_{j} w$ whenever $\left|x_{j}\right|=M$, which would imply $w \in I$. Expanding $w u$ and $v \xi$, and comparing like terms,

$$
v_{j} x_{j}+\sum_{i} v\left(b_{i j} x_{i} x_{j}\right)= \begin{cases}w\left(a_{j} x_{j}\right) & \text { if }\left|x_{j}\right|=M \\ 0 & \text { if }\left|x_{j}\right| \neq M\end{cases}
$$

Thus

$$
v_{j}+v y_{j}= \begin{cases}a_{j} w & \text { if }\left|x_{j}\right|=M \\ 0 & \text { if }\left|x_{j}\right| \neq M\end{cases}
$$


where $y_{j}=\sum_{i} b_{i j} x_{i}$.

Take $j$ so that $\left|x_{j}\right|=M$ and $a_{j} \neq 0$. Since $v_{j}+v y_{j}=a_{j} w, v_{j} \in I$, and $w \notin I$, it follows that $v y_{j} \notin I$. Therefore $v \notin I$.

Since $\xi \neq 0$, there exist $i$ and $j$ such that $b_{i j} x_{i} x_{j} \neq 0$, implying $b_{i j} \neq 0$, and in turn $b_{j i} \neq 0$. If it happens that $\left|x_{j}\right|=M$, we can instead consider the summand $b_{j i} x_{j} x_{i} \neq 0$, where we have $\left|x_{i}\right| \neq M$ since $\left|x_{i}\right|+\left|x_{j}\right|=N$ and $N$ is odd.

In any case, we can choose $i$ and $j$ such that $b_{i j} \neq 0$ and $\left|x_{j}\right| \neq M$. Then $y_{j} \neq 0$, and since $v \neq 0$, $v y_{j} \neq 0$. Now because $v \notin I$ and $v$ is of length $l$, by our inductive assumption $v y_{j} \notin I$. But $v_{j}+v y_{j}=0$ and $v_{j} \in I$, so $v y_{j}=-v_{j}$ is a nonzero element in $I$, a contradiction. Therefore $w u \notin I$, which finishes our induction.

Theorem 3.5. Let $P$ be as in the introduction to this section. Assume the following condition holds true:

$(*)$ there exist elements $a, b \in H^{*}\left(P ; \mathbb{Z}_{p}\right)$ such that $0<|a|<|b|<n,|a|+|b|=n$, and the cup product $a b \in H^{n}\left(P ; \mathbb{Z}_{p}\right)$ is nonzero.

Then there is a Hopf algebra isomorphism

$$
H_{*}\left(\Omega P ; \mathbb{Z}_{p}\right) \cong T\left(\bar{u}_{1}, \ldots, \bar{u}_{\ell}\right) / I,
$$

where $I$ is the two-sided ideal of $H_{*}\left(\Omega \bar{P} ; \mathbb{Z}_{p}\right) \cong T\left(\bar{u}_{1}, \ldots, \bar{u}_{\ell}\right)$ generated by the degree $n-2$ element

$$
\chi=\sum_{m \leq s \leq \ell} b_{s} \kappa_{s}
$$

as described in Proposition 3.2. Moreover, the looped inclusion $\Omega \bar{P} \stackrel{\Omega i}{\longrightarrow} \Omega P$ induces a map on mod-p homology modelled by the canonical map $T\left(\bar{u}_{1}, \ldots, \bar{u}_{\ell}\right) \longrightarrow T\left(\bar{u}_{1}, \ldots, \bar{u}_{\ell}\right) / I$.

Proof. To avoid confusing notation, we will write monomials in $T\left(\bar{u}_{1}, \ldots, \bar{u}_{\ell}\right)$ without the tensor product symbol. By Proposition 3.2 , the element $\chi \in H_{*}\left(\Omega \bar{P} ; \mathbb{Z}_{p}\right) \cong T\left(\bar{u}_{1}, \ldots, \bar{u}_{\ell}\right)$ is in the image of the map

$$
\left(\Omega \alpha^{\prime}\right)_{*}: H_{n-2}\left(S^{n-2} ; \mathbb{Z}_{p}\right) \longrightarrow H_{n-2}\left(\Omega \bar{P} ; \mathbb{Z}_{p}\right)
$$

induced by the adjoint $\alpha^{\prime}$ of the attaching map $\alpha$. Thus $\chi$ is a primitive element, and $(\Omega i)_{*}(\chi)=0$ in $H_{*}\left(\Omega P ; \mathbb{Z}_{p}\right)$, where $i$ is the inclusion $\bar{P} \stackrel{i}{\longrightarrow} P$.

Let $A$ be the quotient algebra of the tensor algebra $T\left(\bar{u}_{1}, \ldots, \bar{u}_{\ell}\right)$ modulo the two-sided ideal generated by the element $\chi$. Then $A$ is a Hopf algebra because $\chi$ is primitive. Since $(\Omega i)_{*}(\chi)=0$ in $H_{*}\left(\Omega P ; \mathbb{Z}_{p}\right)$, the Hopf algebra map $\hat{\theta}=(\Omega i)$ factors through Hopf algebra maps

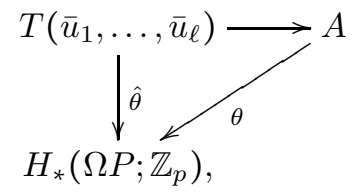

where the Hopf algebra map $\theta$ is defined by $\theta\left(\bar{u}_{i}\right)=u_{i}$.

We let $m^{\prime}$ be the smallest integer $m \leq m^{\prime} \leq\left\lfloor\frac{n}{2}\right\rfloor$ such that there is a $c_{i j}$ prime to $p$ for some $i \leq j$, with $i$ satisfying $\left|a_{i}\right|=m^{\prime}$. By condition $(*)$ such an integer $m^{\prime}$ exists, and $m^{\prime}<n-m^{\prime}$.

Consider differential bigraded left $A$-modules

$$
\hat{E}_{*, \star}^{2}=\cdots=\hat{E}_{*, \star}^{m}=\mathbb{Z}_{p}\left\{1, a_{1}, \ldots, a_{\ell}, z\right\} \otimes A,
$$

the element

$$
\zeta=(-1)^{m^{\prime}} \sum_{\left|a_{i}\right|=m^{\prime},\left|a_{j}\right|=n-m^{\prime}} c_{i j}\left(a_{j} \otimes \bar{u}_{i}\right),
$$

with formal differentials $\hat{d}^{r}$ of bidegree $(-r, r-1)$ for $r \leq m$ given as follows. First define the morphism of left $T\left(\bar{u}_{1}, \ldots, \bar{u}_{\ell}\right)$-modules

$$
\bar{d}^{r}: \mathbb{Z}_{p}\left\{1, a_{1}, \ldots, a_{\ell}, z\right\} \otimes T\left(\bar{u}_{1}, \ldots, \bar{u}_{\ell}\right) \longrightarrow \mathbb{Z}_{p}\left\{1, a_{1}, \ldots, a_{\ell}, z\right\} \otimes T\left(\bar{u}_{1}, \ldots, \bar{u}_{\ell}\right)
$$


by $\bar{d}^{r}=0$ when $r<m$, and respecting the left action of $T\left(\bar{u}_{1}, \ldots, \bar{u}_{\ell}\right)$ by assigning

$$
\bar{d}^{m}(x \otimes y)=(1 \otimes y) \bar{d}^{m}(x \otimes 1),
$$

where $\bar{d}^{m}(1 \otimes y)=0 ; \bar{d}^{m}\left(a_{i} \otimes 1\right)=1 \otimes \bar{u}_{i}$ whenever $\left|a_{i}\right|=m$, otherwise $\bar{d}^{m}\left(a_{i} \otimes 1\right)=0$; and $\bar{d}^{m}(z \otimes 1)=\zeta$ when $m=m^{\prime}$, otherwise $\bar{d}^{m}(z \otimes 1)=0$.

Since $A$ is the quotient of $T\left(\bar{u}_{1}, \ldots, \bar{u}_{\ell}\right)$ subject to the relation $\chi \sim 0$, for $r \leq m$ the differential $\bar{d}^{r}$ induces a morphism $\hat{d}^{r}$ of $A$-modules

$$
\hat{d}^{m}: \mathbb{Z}_{p}\left\{1, a_{1}, \ldots, a_{\ell}, z\right\} \otimes A \longrightarrow \mathbb{Z}_{p}\left\{1, a_{1}, \ldots, a_{\ell}, z\right\} \otimes A
$$

respecting the left action of $A$.

Next we define inductively for $r \geq m$

$$
\hat{E}_{*, *}^{r+1}=\frac{\operatorname{ker}\left(d^{r}: E_{*, *}^{r} \longrightarrow E_{*-r, *+r-1}^{r}\right)}{\operatorname{Im}\left(d^{r}: E_{*+r, *-r+1}^{r} \longrightarrow E_{*, *}^{r}\right)} .
$$

As before, we have formal differentials given as morphisms of left $A$-modules

$$
\hat{d}^{r+1}: \hat{E}_{*, *}^{r+1} \longrightarrow E_{*-(r+1), *+r}^{r+1}
$$

respecting the left action of $A$, and such that: $\hat{d}^{r+1}(1 \otimes y)=0 ; \hat{d}^{r+1}\left(a_{i} \otimes 1\right)=1 \otimes \bar{u}_{i}$ whenever $\left|a_{i}\right|=r+1$, otherwise $\hat{d}^{r+1}\left(a_{i} \otimes 1\right)=0$; and $\hat{d}^{r+1}(z \otimes 1)=\zeta$ if $r+1=m^{\prime}$, otherwise $\hat{d}^{r+1}(z \otimes 1)=0$.

This gives a formal spectral sequence $\hat{E}=\left\{\hat{E}^{r}, \hat{d}^{r}\right\}$. We will need to verify that $\hat{E}_{*, *}^{\infty}=\{0\}$ for $(*, *) \neq(0,0)$, but let us assume that this is the case for now. We shall show by induction that the restiction $\theta: A_{k} \rightarrow H_{k}\left(\Omega P ; \mathbb{Z}_{p}\right)$ of the Hopf algebra map $\theta$ is an isomorphism for each $k$.

Let $E$ be mod-p homology spectral sequence for the path fibration of $P$. The morphism of Hopf algebras $A \stackrel{\theta}{\longrightarrow} H_{*}\left(\Omega P ; \mathbb{Z}_{p}\right)$ induces a morphism of spectral sequences

$$
\theta: \hat{E}_{*, *}^{r} \longrightarrow E_{*, *}^{r}
$$

in the canonical way with $\theta\left(1 \otimes \bar{u}_{i}\right)=1 \otimes u_{i}, \theta\left(\bar{a}_{i} \otimes 1\right)=a_{i} \otimes 1$, and $\theta(z \otimes 1)=z \otimes 1$. Note $\hat{E}_{0, *}^{m} \stackrel{\theta}{\longrightarrow} E_{0, *}^{m}$ is just our map $A \stackrel{\theta}{\longrightarrow} H_{*}\left(\Omega P ; \mathbb{Z}_{p}\right)$, and $A_{q}=H_{q}\left(\Omega P ; \mathbb{Z}_{p}\right)=\{0\}$ for $0<q<m-1$.

Suppose $A_{q} \stackrel{\theta}{\longrightarrow} H_{q}\left(\Omega P ; \mathbb{Z}_{p}\right)$ is an isomorphism for $0<q<k$. This implies $\hat{E}_{0, q}^{r} \stackrel{\theta}{\longrightarrow} E_{0, q}^{r}$ is an isomorphism, and $\hat{E}_{i, q}^{r} \stackrel{\theta}{\longrightarrow} E_{i, q}^{r}$ is an isomorphism when $q+r-1<k$. Since $E_{*, *}^{\infty}=\{0\}$ and $\hat{E}_{*, *}^{\infty}=\{0\}$ when $(*, *) \neq(0,0)$, for some sufficiently large $M>m$ the map $\hat{E}_{0, k}^{M} \stackrel{\theta}{\longrightarrow} E_{0, k}^{M}$ is an isomorphism. By definition of spectral sequences, there is a commutative diagram of short exact sequences

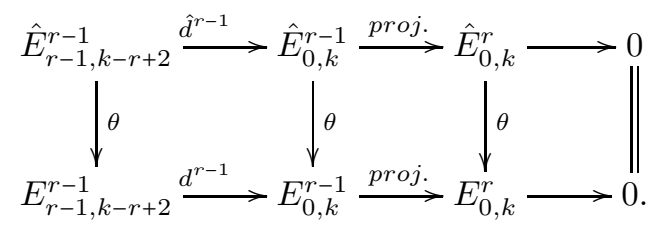

By induction the first vertical map is an isomorphism when $r>2$. When $r=M$ the third vertical map is an isomorphism, and so the second vertical map is also an isomorphism. Iterating this argument over $m \leq r<M$, we see that the map

$$
\theta: A_{k}=\hat{E}_{0, k}^{m} \longrightarrow E_{0, k}^{m}=H_{k}\left(\Omega P ; \mathbb{Z}_{p}\right)
$$

is an isomorphism. This completes the induction.

It remains to check that $\hat{E}_{*, *}^{\infty}=\{0\}$ for $(*, *) \neq(0,0)$. Let $\bar{E}$ be mod- $p$ homology spectral sequence for the path fibration of $\bar{P}$. We have

$$
\bar{E}_{*, *}^{m} \cong \bar{E}_{*, *}^{2}=H_{*}\left(\bar{P} ; \mathbb{Z}_{p}\right) \otimes H_{*}\left(\Omega \bar{P} ; \mathbb{Z}_{p}\right) \cong \mathbb{Z}_{p}\left\{1, a_{1}, \ldots, a_{\ell}\right\} \otimes T\left(\bar{u}_{1}, \ldots, \bar{u}_{\ell}\right),
$$

and $\bar{E}_{*, *}^{\infty}=\{0\}$ when $(*, *) \neq(0,0)$. The Hopf algebra map $H_{*}\left(\Omega \bar{P} ; \mathbb{Z}_{p}\right) \cong T\left(\bar{u}_{1}, \ldots, \bar{u}_{\ell}\right) \longrightarrow A$ induces a morphism of spectral sequences

$$
\phi: \bar{E} \longrightarrow \hat{E}
$$


in the canonical way with $\phi^{2}\left(1 \otimes \bar{u}_{i}\right)=1 \otimes \bar{u}_{i}, \phi^{2}\left(a_{i} \otimes 1\right)=a_{i} \otimes 1$. Notice

$$
\phi^{r}: \bar{E}_{i, j}^{r} \longrightarrow \hat{E}_{i, j}^{r}
$$

is an epimorphism when $i<n$, and is an isomorphism when $i<n, j<n-2$, and $r \leq m^{\prime}$. The differentials $\hat{E}_{i, j}^{r} \stackrel{\hat{d}^{r}}{\longrightarrow} \hat{E}_{i-r, i+r-1}^{r}$ are zero for $r<i$ and $i<n$, so when $i<n$ and $r<\min \{i, n-i\}$, we have projections

$$
\hat{E}_{i, j}^{r} \longrightarrow \hat{E}_{i, j}^{r+1}
$$

that are isomorphisms. Also, $\chi$ is nonzero in $\bar{E}_{0, n-2}^{r}$ for $r \leq n-m^{\prime}$, and zero for $r>n-m^{\prime}$ since $\bar{d}^{n-m^{\prime}}(\zeta)=\chi$.

Note $\hat{E}_{k, l}^{m}=\{0\}$ when $0<k<m, k>n$, or $l<m-1$. We will first consider those nonzero elements in $\hat{E}_{n, l}^{m}$ and $\hat{E}_{0, l}^{m}$ for $l \geq m-1$.

Take any nonzero $x \in \hat{E}_{n, l}^{m}$. Then $x=z \otimes w$ for some nonzero $w \in A$. Note $\hat{d}^{r}\left(\hat{E}_{n, l}^{r}\right)=\{0\}$ when $r<m^{\prime}$, so we can project $x$ to $\hat{E}_{n, l}^{m^{\prime}}$. Pick $w^{\prime} \in T\left(\bar{u}_{1}, \ldots, \bar{u}_{\ell}\right)$ such that $w^{\prime}$ projects onto $w \in A$. Since $w$ is nonzero in $A, w^{\prime}$ is not in the two-sided ideal generated by $\chi$. Take the element following element in $T\left(\bar{u}_{1}, \ldots, \bar{u}_{\ell}\right)$ :

$$
\sigma_{j}^{\prime}=w\left(\sum_{\left|a_{i}\right|=m^{\prime}} c_{i j} \bar{u}_{i}\right) .
$$

Let $\sigma_{j} \in A$ be the projection of $\sigma_{j}^{\prime}$ onto $A$. We have

$$
\begin{aligned}
\hat{d}^{m^{\prime}}(x) & =(1 \otimes w) \hat{d}^{m^{\prime}}(z \otimes 1)=(1 \otimes w)(\zeta) \\
& =(-1)^{m^{\prime}} \sum_{\left|a_{i}\right|=m^{\prime},\left|a_{j}\right|=n-m^{\prime}} c_{i j}\left(a_{j} \otimes\left(w \bar{u}_{i}\right)\right) \\
& =(-1)^{m^{\prime}} \sum_{\left|a_{j}\right|=n-m^{\prime}} a_{j} \otimes \sigma_{j} .
\end{aligned}
$$

By condition $(*)$ there are integers $k<l$, with $\left|a_{l}\right|=m^{\prime}$ and $\left|a_{k}\right|=n-m^{\prime}$, such that $c_{l k}$ is prime to $p$, so the element $\sigma_{k}^{\prime}$ is nonzero. Because $n$ is odd and $w^{\prime}$ is not in the two-sided ideal generated by $\chi$, Lemma 3.4 implies $\sigma_{k}^{\prime}$ is not in the two-sided ideal generated by $\chi$. Therefore $\sigma_{k} \in A$ is nonzero, so $a_{k} \otimes \sigma_{k} \in \hat{E}_{*, \star}^{m}=\mathbb{Z}_{p}\left\{1, a_{1}, \ldots, a_{\ell}, z\right\} \otimes A$ is nonzero, and by this we see that $\hat{d}^{m^{\prime}}(x) \in \hat{E}_{n-m^{\prime}, l+m^{\prime}-1}^{m}$ is also nonzero. By the projection isomorphisms (13), this implies $\hat{d}^{m^{\prime}}(x) \in \hat{E}_{n-m^{\prime}, l+m^{\prime}-1}^{m^{\prime}}$ is nonzero, and so $x$ does not survive to $\hat{E}_{n, l}^{m^{\prime}+1}$. Thus $\hat{E}_{n, l}^{\infty}=\hat{E}_{n, l}^{m^{\prime}+1}=\{0\}$.

Now take $x \in \hat{E}_{0, l}^{m}$ for $l \geq m-1$. We can pick $x^{\prime} \in \bar{E}_{0, l}^{m}$ so that $\phi^{m}\left(x^{\prime}\right)=x$. Since $\bar{E}_{0, l}^{\infty}=\{0\}$, there exists an $\dot{x} \in \bar{E}_{*, *}^{r}$ for some $r \geq m$ such that $\bar{d}^{r}(\dot{x})=x^{\prime}$. Then in $\hat{E}_{0, l}^{r}$,

$$
x=\phi^{r}\left(x^{\prime}\right)=\phi^{r}\left(\bar{d}^{r}(\dot{x})\right)=\hat{d}^{r}\left(\phi^{r}(\dot{x})\right),
$$

and so $x=0$ in $\hat{E}_{0, l}^{r+1}$. Thus $\hat{E}_{0, l}^{\infty}=\{0\}$.

It remains to consider those elements in $\hat{E}_{k, l}^{m}$ when $m \leq k<n$. Because the elements in $\hat{E}_{k, 0}^{m}$ for $m \leq k<n$ are transgressive, the differentials $\hat{E}_{k, l}^{i} \stackrel{d^{i}}{\longrightarrow} \hat{E}_{k-i, l+i-1}^{i}$ are zero for $l \geq 0$ and $m \leq i<k$, and so we might as well project to $\hat{E}_{k, l}^{k}$.

Suppose $x \in \hat{E}_{k, l}^{k}$ and $x \neq 0$. We will show that $\hat{d}^{k}(x) \neq 0$. Hence $\hat{E}_{k, l}^{\infty}=\hat{E}_{k, l}^{k+1}=\{0\}$. There are three subcases: $m \leq k<n-m^{\prime}, k=n-m^{\prime}$, and $n-m^{\prime}<k<n$. We assume $x \neq 0$ and $\hat{d}^{k}(x)=0$ to arrive at a contradiction.

Let us first consider the case $m \leq k<n-m^{\prime}$. We can pick $x^{\prime} \in \bar{E}_{k, l}^{k}$ such that $\phi^{k}\left(x^{\prime}\right)=x$. Then $\phi^{k}\left(\bar{d}^{k}\left(x^{\prime}\right)\right)=\hat{d}^{k}(x)=0$, and so inspecting the kernel of $\bar{E}_{0, k+l-1}^{k} \stackrel{\phi^{k}}{\longrightarrow} \hat{E}_{0, k+l-1}^{k}, y^{\prime}=\bar{d}^{k}\left(x^{\prime}\right) \in \bar{E}_{0, k+l-1}^{k}$ must be a linear combination

$$
y^{\prime}=\sum_{i} v_{i} \chi w_{i}
$$


where $v_{i}$ and $w_{i}$ are monomials in $T\left(\bar{u}_{1}, \ldots, \bar{u}_{\ell}\right)$. Since $x^{\prime}$ is nonzero in $\bar{E}_{*, *}^{k}$, and $\bar{E}_{*, *}^{\infty}=\{0\}$ for $(*, *) \neq(0,0), y^{\prime}$ must also nonzero in $\bar{E}_{*, *}^{k}$, and so we might as well assume the monomials $v_{i}$ and $w_{i}$ are nonzero. Since $y^{\prime}$ is in the image of $\bar{d}^{k}$ with $k<n-m^{\prime}$, and (by definition of $m^{\prime}$ ) $\chi$ is linear combination containing summands $c_{i j} u_{i} u_{j}$ with $\left|u_{j}\right|=n-m^{\prime}$ and $c_{i j}$ prime to $p$, each of the $w_{i}$ 's must be a monomial of length at least 1 of the form

$$
w_{i}=w_{i}^{\prime} \bar{u}_{k_{i}}
$$

for some monomial $w_{i}^{\prime}$ and $\bar{u}_{k_{i}}$ such that $\left|\bar{u}_{k_{i}}\right|=k$. Since $\bar{d}^{k}\left(x^{\prime}\right)=y^{\prime}$,

$$
x^{\prime}=\sum_{i} a_{k_{i}} \otimes v_{i} \chi w_{i}^{\prime} .
$$

But since $\chi$ is zero in $A$, each term $v_{i} \chi w_{i}^{\prime}$ is as well, and so $x=0$ in $\hat{E}_{k, l}^{k}$, a contradiction. Hence we must have $\hat{d}^{k}(x) \neq 0$.

Now consider the case $n-m^{\prime}<k<n$. As in the previous case, we can write $y^{\prime}$ as

$$
y^{\prime}=\sum_{i} v_{i} \chi w_{i}
$$

Here $w \chi$ is also zero in $\bar{E}_{*, *}^{k}$ for any monomial $w$, since

$$
w \chi=\bar{d}^{n-m^{\prime}}\left((-1)^{m^{\prime}} \sum_{\left|a_{i}\right|=m^{\prime},\left|a_{j}\right|=n-m^{\prime}} c_{i j}\left(a_{j} \otimes w \bar{u}_{i}\right)\right)
$$

Therefore each monomial $w_{i}$ is nonzero of length at least 1 , (for otherwise $0=y^{\prime}=\bar{d}^{k}\left(x^{\prime}\right)$, which implies $\left.x^{\prime}=0\right)$, with $w_{i}=w_{i}^{\prime} \bar{u}_{k_{i}}$ for some monomial $w_{i}^{\prime}$ and $\bar{u}_{k_{i}}$ such that $\left|\bar{u}_{k_{i}}\right|=k$. As before this implies $x=0$, a contradiction. Thus $\hat{d}^{k}(x) \neq 0$.

Finally let us consider $k=n-m^{\prime}$. In this case we can write

$$
y^{\prime}=\sum_{i} v_{i} \chi w_{i}+\sum_{i} y_{i} \chi
$$

for some nonzero monomial $v_{i}$, and nonzero monomial $w_{i}$ of length at least 1 . As before, we must have $w_{i}=w_{i}^{\prime} \bar{u}_{k_{i}}$ for some $w_{i}^{\prime}$ and $\bar{u}_{k_{i}}$ such that $\left|\bar{u}_{k_{i}}\right|=n-m^{\prime}$. Let $\zeta^{\prime} \in \bar{E}_{n-m^{\prime}, m^{\prime}-1}^{n-m^{\prime}}$ be the element satisfying $\phi^{n-m^{\prime}}\left(\zeta^{\prime}\right)=\zeta$. Notice that in $\bar{E}_{0, n-2}^{n-m^{\prime}}$ we have $\bar{d}^{n-m^{\prime}}\left(b \zeta^{\prime}\right)=\chi$ for some integer $b$ prime to $p$. Thus

Since $\chi$ is zero in $A$,

$$
x^{\prime}=\sum_{i}\left(a_{k_{i}} \otimes v_{i} \chi w_{i}^{\prime}\right)+b \sum_{i}\left(1 \otimes y_{i}\right) \zeta^{\prime} .
$$

$$
x=\phi^{n-m^{\prime}}\left(x^{\prime}\right)=\phi^{n-m^{\prime}}\left(b \sum_{i}\left(1 \otimes y_{i}\right) \zeta^{\prime}\right)=b \sum_{i}\left(1 \otimes y_{i}\right) \zeta .
$$

But in $\hat{E}_{*, *}^{m^{\prime}}$ we have $\bar{d}^{m^{\prime}}(z \otimes 1)=\zeta$, so $\bar{d}^{m^{\prime}}\left(z \otimes y_{i}\right)=\left(1 \otimes y_{i}\right) \zeta$. So because $m^{\prime}<n-m^{\prime},\left(1 \otimes y_{i}\right) \zeta$ is zero in $\hat{E}_{*, *}^{n-m^{\prime}}$, and it follows that $x=0$, a contradiction. Hence $\hat{d}^{k}(x) \neq 0$.

\section{Some additional structure on the mod- $p$ Cohomology Ring}

The non-trivial action of Bockstein operations impose the restrictions seen in Proposition 2.1] Later we will see they are necessary for Theorem 2.3 to be true in general. Recall that the mod- $p$ Bockstein operations $\beta_{r}: H_{*}(X) \longrightarrow H_{*-1}(X)$ are derivations with respect to the homology multiplication induced by an $H$-space structure on $X$. That is,

$$
\beta_{r}(x y)=\beta_{r}(x) y+(-1)^{|x|} x \beta_{r}(y) .
$$

Proof of Proposition 2.1. Take the attaching map $S^{2 n-2} \stackrel{\alpha}{\longrightarrow} \bar{W}$ and its adjoint $\alpha^{\prime}$. By Proposition 3.2 in $H_{2 n-3}\left(\Omega \bar{W} ; \mathbb{Z}_{p}\right)$ we have

$$
\alpha_{\star}^{\prime}\left(\iota_{2 n-3}\right)=\sum_{i, j}(-1)^{n-1} a_{i j}\left[\bar{u}_{i}, \bar{v}_{j}\right] .
$$


for some generator $\iota_{2 n-3} \in H_{2 n-3}\left(S^{2 n-3} ; \mathbb{Z}_{p}\right)$. As sets we have

$$
\left\{r_{1}, r_{2}, \ldots, r_{k_{1}}\right\}=\left\{s_{1}, s_{2}, \ldots, s_{l}\right\}
$$

for some $s_{1}<s_{2}<\cdots<s_{l}$ and $l \leq k_{1}$. Since $\bar{u}_{i}$ and $\bar{v}_{i}$ are the transgressions of $x_{i}$ and $y_{i}, \beta_{r_{i}}\left(\bar{v}_{i}\right)=\bar{u}_{i}$ for $1 \leq i \leq k_{1}$, and $\beta_{r}\left(\bar{v}_{i}\right)=0$ for each $r>0$ and $k_{1}<i \leq k$. Therefore $\beta_{s_{t}}\left(\left[\bar{u}_{i}, \bar{v}_{j}\right]\right)=0$ whenever $j>k_{1}$ or $s_{t} \neq r_{j}$. Then because $\beta_{s_{t}}\left(\alpha_{*}^{\prime}\left(\iota_{2 n-3}\right)\right)=\alpha_{*}^{\prime}\left(\beta_{s_{t}}\left(\iota_{2 n-3}\right)\right)=\alpha_{*}^{\prime}(0)=0$ for each $t$,

$$
\begin{aligned}
0 & =\sum_{t=1}^{l} \beta_{s_{t}}\left(\alpha_{*}^{\prime}\left(\iota_{2 n-3}\right)\right) \\
& =\sum_{t=1}^{l} \beta_{s_{t}}\left(\sum_{i, j}(-1)^{n-1} a_{i j}\left[\bar{u}_{i}, \bar{v}_{j}\right]\right) \\
& =\sum_{j \leq k_{1}, i}(-1)^{n-1} a_{i j} \beta_{r_{j}}\left(\left[\bar{u}_{i}, \bar{v}_{j}\right]\right) \\
& =(-1)^{n-1} \sum_{j \leq k_{1}, i} a_{i j}\left[\bar{u}_{i}, \bar{u}_{j}\right] \\
& =(-1)^{n-1}\left(\sum_{i=1}^{k_{1}} a_{i i}\left[\bar{u}_{i}, \bar{u}_{i}\right]+\sum_{j<i \leq k_{1}}\left(a_{i j}-(-1)^{n} a_{j i}\right)\left[\bar{u}_{i}, \bar{u}_{j}\right]+\sum_{j \leq k_{1}, i>k_{1}} a_{i j}\left[\bar{u}_{i}, \bar{u}_{j}\right]\right) .
\end{aligned}
$$

When $n$ is odd it follows that $a_{i i}=0$ and $a_{i j}+a_{j i}=0$ whenever $j<i \leq k_{1}$, and $a_{i j}=0$ whenever $1 \leq j \leq k_{1}$ and $k_{1}<i \leq k$. Namely $B_{z^{*}}$ is skew symmetric and $C_{z^{*}}=0$. When $n$ is even $a_{i j}-a_{j i}=0$ and $\left[\bar{u}_{i}, \bar{u}_{i}\right]=0$, so there is no restriction on the $a_{i i}$ 's. In this case $B_{z^{*}}$ is symmetric, and likewise $C_{z^{*}}=0$.

\section{The Effect of Looping in Rank One}

Fix a class $[V] \in \mathcal{T}_{1, n}^{p}$ for $p$ an odd prime. Again we assume that all our spaces are localized at p. $H_{*}\left(V ; \mathbb{Z}_{p}\right)$ is generated by $x, y$, and $z$, where $|x|=n-1,|y|=n$, and $|z|=2 n-1$. If $\beta_{r}(y)=x$ for some $r>0$, then we can and will take $V \in[V]$ so that $(2 n-2)$-skeleton $\bar{V}$ of $V$ is the Moore space space $P^{n}\left(p^{r}\right)$. Similarly, when $\beta_{r}(y)=0$ for every $r>0, V \in[V]$ can be taken so that $\bar{V}=S^{n-1} \vee S^{n}$.

Let $u$ and $v$ in $H_{*}\left(\Omega V ; \mathbb{Z}_{p}\right)$ be the transgressions of $x$ and $y$ respectively, with $|v|=n-1$ and $|u|=n-2$. The following are consequences of Proposition 3.5 and Proposition 3.2.

Corollary 5.1. Take $[V] \in \mathcal{T}_{1, n}^{p}$ with $n \geq 3$. Then $H_{*}\left(\Omega V ; \mathbb{Z}_{p}\right) \cong T(u) \otimes T(v)$ as Hopf algebras.

Corollary 5.2. Take $[V] \in \mathcal{T}_{1, n}^{p}$ with $n \geq 3$. Let $\alpha: S^{2 n-2} \rightarrow P^{n}\left(p^{r}\right)$ be the attaching map for the $V$, and $\alpha^{\prime}: S^{2 n-3} \rightarrow \Omega P^{n}\left(p^{r}\right)$ be the adjoint map of $\alpha$. Then

$$
\alpha_{*}^{\prime}\left(\iota_{2 n-3}\right)=[u, v] .
$$

The following lemma is a special case of Barratt's work on growth of homotopy exponents [2], or Theorem 4.1 in [6].

Proposition 5.3. Fix some $n \geq 4$, and let $C$ be a finite wedge of Moore spaces $P^{n}\left(p^{r_{i}}\right)$. Let $s=\max _{i}\left\{r_{i}\right\}$. Then

$$
p^{s+k} \pi_{i}(C)=0 \text { if } i \leq p^{k+1}(n-2) .
$$

Let $S^{2 m-1}\left\{p^{r}\right\}$ be the homotopy theoretic fiber of the degree $p^{r}$ map $S^{2 m-1} \stackrel{p^{r}}{\longrightarrow} S^{2 m-1}$. Recall from [4] there exists map

$$
h: S^{2 m-1}\left\{p^{r}\right\} \stackrel{h}{\longrightarrow} \Omega P^{2 m}\left(p^{r}\right)
$$

that is modelled on mod- $p$ homology by mapping $H_{*}\left(S^{2 m-1}\left\{p^{r}\right\} ; \mathbb{Z}_{p}\right)$ isomorphically onto the left

$T(u)$-submodule of $H_{*}\left(\Omega P^{2 m}\left(p^{r}\right) ; \mathbb{Z}_{p}\right) \cong T(u, v)$. This map has a left homotopy inverse $\Omega P^{2 m}\left(p^{r}\right) \stackrel{h^{-1}}{\longrightarrow}$ $S^{2 m-1}\left\{p^{r}\right\}$ that induces a map on mod- $p$ homology modelled by the abelianization map $T(u, v) \longrightarrow$ $S(u, v)$. 
Lemma 5.4. Let $[V] \in \mathcal{T}_{1,2 m}^{p}$ with $m>2$ and $\beta_{r}(y)=x$ for some $r>0$. Then $v^{2}$ is spherical in $H_{*}\left(\Omega V ; \mathbb{Z}_{p}\right)$.

Proof. Let $S^{4 m-2} \stackrel{\alpha}{\longrightarrow} P^{2 m}\left(p^{r}\right)$ be the attaching map for $V$. By Proposition [5.3, $[\alpha]$ has order $p^{r}$ in $\pi_{4 m-2}\left(P^{2 m}\left(p^{r}\right)\right)$. Thus $\alpha$ extends to a map $P^{4 m-1}\left(p^{r}\right) \stackrel{\bar{\alpha}}{\longrightarrow} P^{2 m}\left(p^{r}\right)$. By taking the adjoint of $\bar{\alpha}$, we have the map

$$
\bar{\alpha}^{\prime}: P^{4 m-2}\left(p^{r}\right) \longrightarrow \Omega P^{2 m}\left(p^{r}\right)
$$

which induces

$$
\bar{\alpha}_{*}^{\prime}: \bar{H}_{*}\left(P^{4 m-2}\left(p^{r}\right) ; \mathbb{Z}_{p}\right) \longrightarrow H_{*}\left(\Omega P^{2 m}\left(p^{r}\right) ; \mathbb{Z}_{p}\right) .
$$

Let $u^{\prime} \in H_{4 m-3}\left(P^{4 m-2}\left(p^{r}\right) ; \mathbb{Z}_{p}\right)$ and $v^{\prime} \in H_{4 m-2}\left(P^{4 m-2}\left(p^{r}\right) ; \mathbb{Z}_{p}\right)$ be a basis with $\beta_{r}\left(v^{\prime}\right)=u^{\prime}$. Since $\bar{\alpha}^{\prime}$ restricted to $S^{4 m-3}$ is $\alpha^{\prime}$, we have

$$
\bar{\alpha}_{\star}^{\prime}\left(u^{\prime}\right)= \pm[u, v] .
$$

We may assume that $\bar{\alpha}_{*}^{\prime}\left(u^{\prime}\right)=[u, v]$ because otherwise we can replace $u^{\prime}$ and $v^{\prime}$ to be $-u^{\prime}$ and $-v^{\prime}$.

When $m>2,\left|u^{3}\right|=3(2 m-2)=6 m-6>\left|v^{2}\right|=2(2 m-1)=4 m-2$, which implies $2 m>4$. Then $H_{4 m-2}\left(\Omega P^{2 m}\left(p^{r}\right) ; \mathbb{Z}_{p}\right)$ is a 1 -dimensional vector space with a basis given by $v^{2}$, implying $\alpha_{*}^{\prime}\left(v^{\prime}\right)=k v^{2}$ for some $k$. Since

$$
k[u, v]=\beta_{r}\left(k v^{2}\right)=\beta_{r}\left(\bar{\alpha}_{*}^{\prime}\left(v^{\prime}\right)\right)=\bar{\alpha}_{*}^{\prime}\left(\beta_{r}\left(v^{\prime}\right)\right)=\bar{\alpha}_{*}^{\prime}\left(u^{\prime}\right)=[u, v],
$$

we have $k=1$. Therefore $\bar{\alpha}_{*}^{\prime}\left(v^{\prime}\right)=v^{2}$.

Consider the composite

$$
f: P^{4 m-2}\left(p^{r}\right) \stackrel{\bar{\alpha}^{\prime}}{\longrightarrow} \Omega P^{2 m}\left(p^{r}\right) \longrightarrow \Omega V .
$$

By Corollary 5.1 there is a Hopf algebra isomorphism $H_{*}\left(\Omega V ; \mathbb{Z}_{p}\right) \cong T(u) \otimes T(v)$, and the $H$-map $\Omega P^{2 m}\left(p^{r}\right) \longrightarrow \Omega V$ induces a map on mod- $p$ homology modelled by the algebra map $T(u, v) \longrightarrow$ $T(u) \otimes T(v)$ sending $u$ to $u$ and $v$ to $v$. Thus $f_{*}\left(\iota_{4 m-2}\right)=v^{2}$.

Now $f$ factors through a map

$$
\bar{f}: S^{4 m-2} \longrightarrow \Omega V,
$$

because its restriction to the bottom sphere $S^{4 m-3}$ is the adjoint of the null homotopic map $S^{4 m-2} \stackrel{\alpha}{\longrightarrow}$ $P^{2 m}\left(p^{r}\right) \longrightarrow V$, and we have $\bar{f}_{*}\left(\iota_{4 m-2}\right)=f_{*}\left(\iota_{4 m-2}\right)=v^{2}$.

Theorem 5.5. Take $[V] \in \mathcal{T}_{1,2 m}^{p}$ with $m>2$ and $\beta_{r}(y)=x$ for some $r>0$. Then

$$
\Omega V \simeq S^{2 m-1}\left\{p^{r}\right\} \times \Omega S^{4 m-1} .
$$

Proof. Consider the composite

$$
\phi: S^{2 m-1}\left\{p^{r}\right\} \stackrel{h}{\longrightarrow} \Omega P^{2 m}\left(p^{r}\right) \longrightarrow \Omega V,
$$

where the last map is the looped inclusion. The map $S^{2 m-1}\left\{p^{r}\right\} \stackrel{h}{\longrightarrow} \Omega P^{2 m}\left(p^{r}\right)$ is modelled on mod- $p$ homology by mapping $H_{*}\left(S^{2 m-1}\left\{p^{r}\right\} ; \mathbb{Z}_{p}\right)$ isomorphically onto the left $T(u)$-submodule of $H_{*}\left(\Omega P^{2 m}\left(p^{r}\right) ; \mathbb{Z}_{p}\right) \cong T(u, v)$ with basis $\{1, v\}$, where $|v|=n-1$ and $|u|=n-2$. Also, by Theorem 3.5, there is a Hopf algebra isomorphism

$$
H_{\star}\left(\Omega V ; \mathbb{Z}_{p}\right) \cong T(u) \otimes T(v)
$$

and the $H$-map $\Omega P^{2 m}\left(p^{r}\right) \longrightarrow \Omega V$ induces a map on mod-p homology modelled by the algebra map $T(u, v) \longrightarrow T(u) \otimes T(v)$ sending $u$ to $u$ and $v$ to $v$. It follows that $\phi_{*}$ is modelled by an isomorpism onto the left $T(u)$-submodule of $T(u) \otimes T(v)$ with basis $\{1, v\}$.

Now consider the map $S^{4 m-2} \stackrel{\alpha^{\prime}}{\longrightarrow} \Omega V$ from the proof of Lemma 5.4 which makes the class $v^{2} \epsilon$ $H_{*}\left(\Omega V ; \mathbb{Z}_{p}\right)$ spherical. Since $\Omega V$ is an $H$-space, by the universal property of the James construction for $\Omega S^{4 m-1} \alpha^{\prime}$ extends to an $H$-map $\Omega S^{4 m-1} \stackrel{\theta}{\longrightarrow} \Omega V$. Then $\theta_{\star}$ is modelled on mod- $p$ homology by mapping $T\left(\iota_{4 m-2}\right)$ onto the subalgebra of $T(u) \otimes T(v)$ generated by $v^{2}$.

One now sees that the product

$$
S^{2 m-1}\left\{p^{r}\right\} \times \Omega S^{4 m-1} \stackrel{\phi \times \theta}{\longrightarrow} \Omega V \times \Omega V \stackrel{\text { mult. }}{\longrightarrow} \Omega V
$$

induces an isomorphism on mod- $p$ homology, thus is a homotopy equivalence. 
The following theorem is probably well known.

Theorem 5.6. Take $[V] \in \mathcal{T}_{1, n}^{p}$ with $\beta_{r}(y)=0$ for each $r>0$. Then

$$
\Omega V \simeq \Omega S^{n-1} \times \Omega S^{n} .
$$

Proof. We take $V \in[V]$ so that $\bar{V}=S^{n-1} \vee S^{n}$. Recall for general spaces $X$ and $Y$, the looped inclusion $\Omega(X \vee Y) \longrightarrow \Omega(X \times Y)=\Omega X \times \Omega Y$ has a right homotopy inverse. Thus for $X=S^{n-1}$ and $Y=S^{n}$ we have a right homotopy inverse

$$
\Omega S^{n-1} \times \Omega S^{n} \stackrel{s}{\longrightarrow} \Omega\left(S^{n-1} \vee S^{n}\right) .
$$

On mod- $p$ homology, $s_{\star}$ is modelled by the inclusion of Hopf algebras $T\left(u^{\prime}\right) \otimes T\left(v^{\prime}\right) \longrightarrow T\left(u^{\prime}, v^{\prime}\right)$, where $\left|x^{\prime}\right|=n-2$ and $\left|y^{\prime}\right|=n-1$. Since $\Omega\left(i^{\prime}\right)_{*}$ is an algebra map, it is clear that the composite

$$
\Omega S^{n-1} \times \Omega S^{n} \stackrel{s}{\longrightarrow} \Omega\left(S^{n-1} \vee S^{n}\right) \stackrel{i}{\longrightarrow} \Omega V
$$

induces an isomorphism in mod- $p$ homology, and so is a homotopy equivalence.

\section{Higher RANKS}

Throughout this section we fix some class $[W] \in \mathcal{T}_{k, 2 m}^{p}$ with $k \geq 2$, and $m>2$, with all spaces localized at an odd prime $p$.

We recall the properties for $W$ described in Section 2 . The generators $x_{1}, \ldots, x_{k}$ and $y_{1}, \ldots, y_{k}$ will denote the basis for $H_{2 m-1}\left(W ; \mathbb{Z}_{p}\right)$ and $H_{2 m}\left(W ; \mathbb{Z}_{p}\right)$ dual to the mod- $p$ cohomology basis that we gave earlier, while $u_{1}, \ldots, u_{k} \in H_{2 m-2}\left(\Omega V ; \mathbb{Z}_{p}\right)$ and $v_{1}, \ldots, v_{k} \in H_{2 m-1}\left(\Omega V ; \mathbb{Z}_{p}\right)$ will denote the transgressions of the $x_{i}$ 's and $y_{j}$ 's. We have $\beta_{r_{i}}\left(y_{i}\right)=x_{i}$ for some integers $r_{1}, \ldots, r_{k_{1}}$, and integer $0 \leq k_{1} \leq k$. For convenience we take $W \in[W]$ so that the homotopy equivalence in equation (2), corresponding to our choice of basis above, is a homeomorphism.

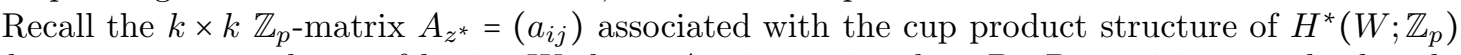
with respect to our choice of basis. We have $A_{z^{*}}$ is nonsingular. By Proposition 2.1 the $k_{1} \times k_{1}$ matrix $B_{z^{*}}$ in the block partition of $A_{z^{*}}$ (equation [3) is symmetric, and the $k_{2} \times k_{1}$ matrix $C_{z^{*}}$ is zero. In paricular $a_{i j}=0$ for $k_{1}<i \leq k$, and $a_{i j}=a_{j i}$ whenever $1 \leq i \leq k_{1}$.

Let us assume $k_{1} \geq 1$ for now. We may as well assume our mod- $p$ homology basis has been ordered so that

$$
r_{1}=\max \left\{r_{1}, \ldots, r_{k_{1}}\right\} .
$$

Since $A_{z^{*}}$ is nonsingular, there must exist an integer $i>1$ such that $a_{i 1} \neq 0$ whenever $a_{11}=0$. If this is the case, for convenience we assume our mod- $p$ homology basis corresponding to the splitting of $\bar{W}$ has been ordered so that $i=2$.

We will construct a certain map

$$
q: W \longrightarrow V
$$

which will be used in the upcoming proofs. Here $[V] \in \mathcal{T}_{1,2 m}^{p}$ with $\bar{V}=P^{2 m}\left(p^{r_{1}}\right)$, and $q_{*}$ is nonzero in degree $2 n-1$, and is nonzero for some choice of degree $2 m-1$ and degree $2 m$ generators. The restrictions on the matrix $A_{z^{*}}$ mentioned above will be necessary in order for $q$ to exist in general. A similar map is constructed for the special case $k_{1}=0$. This construction of will depend on a few seperate cases, again assuming $k_{1} \geq 1$ :

(1) $a_{11} \neq 0$.

(2) $a_{11}=0$ : Since $A_{z^{*}}$ is nonsingular, there is an integer $i>1$ such that $a_{i 1} \neq 0$. We must have $i \leq k_{1}$, because $a_{i j}=0$ when $i>k_{1}$. So $i$ corresponds to a Moore space $P^{2 m}\left(p^{r_{i}}\right)$ in the splitting of $\bar{W}$. We consider three subcases:

(a) $r_{1}=r_{2}$ and $a_{22} \neq 0$;

(b) $r_{1}=r_{2}$ and $a_{22}=0$;

(c) $r_{1}>r_{2}$. 
If the first case holds, let $\hat{W}=\bar{W} / P^{2 m}\left(p^{r_{1}}\right)$. One may notice that the quotient $V=W / \hat{W}$, which extends the quotient $\bar{W} / \hat{W}=P^{2 m}\left(p^{r_{1}}\right)$, has its homotopy type in $\mathcal{T}_{1,2 m}^{p}$. Otherwise when part $(a)$ of the second case holds, let us fix $\hat{W}=\bar{W} / P^{2 m}\left(p^{r_{2}}\right)$ and $V=W / \hat{W}$. In either case we set $W \stackrel{q}{\longrightarrow} V$ as the respective quotient map.

Now consider parts $(b)$ and $(c)$ of the second case. $A_{z^{*}}$ being symmetric implies $a_{21}=a_{12}$. Setting $\hat{W}=\bar{W} /\left(P^{2 m}\left(p^{r_{1}}\right) \vee P^{2 m}\left(p^{r_{2}}\right)\right)$, let $V^{\prime}$ denote the quotient $W / \hat{W}$ and $W \stackrel{q^{\prime}}{\rightarrow} V^{\prime}$ the corresponding quotient map. Set $t=r_{1}-r_{2} \geq 0$, and take the map $P^{2 m}\left(p^{r_{2}}\right) \stackrel{\zeta}{\longrightarrow} P^{2 m}\left(p^{r_{1}}\right)$ as the induced map of cofibers in the cofibration diagram

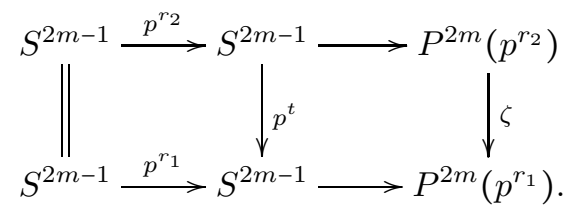

Let $V$ be the pushout in the pushout diagram

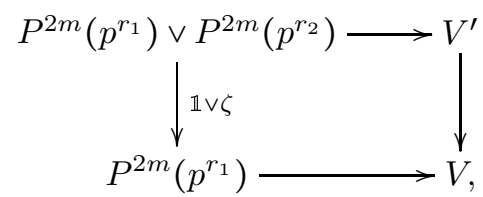

where the horizontal maps are inclusions. Let $q$ be the composite

$$
q: W \stackrel{q^{\prime}}{\longrightarrow} V^{\prime} \longrightarrow V .
$$

Notice $q$ extends the composite

$$
\bar{W} \longrightarrow \bar{W} / \hat{W}=P^{2 m}\left(p^{r_{1}}\right) \vee P^{2 m}\left(p^{r_{2}}\right) \stackrel{\mathbb{1} \vee \zeta}{\longrightarrow} P^{2 m}\left(p^{r_{1}}\right),
$$

and $H^{n}\left(P^{2 m}\left(p^{r_{2}}\right) ; \mathbb{Z}_{p}\right) \stackrel{\zeta^{*}}{\longrightarrow} H^{n}\left(P^{2 m}\left(p^{r_{1}}\right) ; \mathbb{Z}_{p}\right)$ is an isomorphism when $n=2 m$, and multiplication by $p^{t}$ when $n=2 m-1$ (hence trivial when $t>0$ ). Thus

$$
q^{*}\left(x^{*}\right)=x_{1}^{*}+p^{t} x_{2}^{*},
$$

and

$$
q^{*}\left(y^{*}\right)=y_{1}^{*}+y_{2}^{*}
$$

for some generators $x^{*}$ and $y^{*}$ in $H^{2 m-1}\left(V ; \mathbb{Z}_{p}\right)$ and $H^{2 m}\left(V ; \mathbb{Z}_{p}\right)$. For part $(c)$, when $t=r_{1}-r_{2}>0$, we have

$$
q^{*}\left(x^{*} y^{*}\right)=\left(x_{1}^{*}+p^{t} x_{2}^{*}\right)\left(y_{1}^{*}+y_{2}^{*}\right)=\left(a_{11}+a_{21}+p^{t} a_{12}+p^{t} a_{22}\right) z^{*}=a_{21} z^{*} .
$$

Therefore $x^{*} y^{*}=a_{21} e^{*}$ for some generator $e \in H^{4 m-1}\left(V ; \mathbb{Z}_{p}\right) \cong \mathbb{Z}_{p}$. Since we are assuming $a_{21} \neq 0$, the homotopy type of $V$ is in $\mathcal{T}_{1,2 m}^{p}$. For part $(b)$, when $t=r_{1}-r_{2}=0$ and $a_{22}=0$,

$$
q^{*}\left(x^{*} y^{*}\right)=\left(a_{21}+a_{12}\right) z^{*}=2\left(a_{21}\right) z^{*},
$$

and so the homotopy type of $V$ is in $\mathcal{T}_{1,2 m}^{p}$ for this case as well.

Finally we consider the construction of the map $W \stackrel{q}{\longrightarrow} V$ for the case $k_{1}=0$. This time $[V] \in \mathcal{T}_{k, 2 m}^{p}$ satisfies $\bar{V}=S^{n-1} \vee S^{n}$. The construction is straightforward. The nonsingular $A_{z^{*}}$ must have $a_{i 1} \neq 0$ for some $i$. Assume our basis has been ordered so that $i=1$. Let $\hat{W}=\bar{W} /\left(S^{n-1} \vee S^{n}\right)$, where the spheres $S^{n-1}$ and $S^{n}$ in the splitting of $\bar{W}$ correspond to the generators $x_{1}$ and $y_{1}$. Now let $V=W / \hat{W}$, and $q$ be the corresponding quotient map.

We shall let $x^{*} \in H^{2 m-1}\left(\bar{V} ; \mathbb{Z}_{p}\right)=H^{2 m-1}\left(V ; \mathbb{Z}_{p}\right)$ and $y^{*} \in H^{2 m}\left(\bar{V} ; \mathbb{Z}_{p}\right)=H^{2 m}\left(V ; \mathbb{Z}_{p}\right)$ be generators with $\beta_{r}\left(x^{*}\right)=y^{*}, x \in H_{2 m-1}\left(\bar{V} ; \mathbb{Z}_{p}\right)=H_{2 m-1}\left(V ; \mathbb{Z}_{p}\right)$ and $y \in H_{2 m}\left(\bar{V} ; \mathbb{Z}_{p}\right)=H_{2 m}\left(V ; \mathbb{Z}_{p}\right)$ be their homology duals, and $u \in H^{2 m-2}\left(\Omega \bar{V} ; \mathbb{Z}_{p}\right)$ and $v \in H^{2 m-1}\left(\Omega \bar{V} ; \mathbb{Z}_{p}\right)$ be the transgressions of $x$ and $y$.

The following lemma can be viewed as an extension of Lemma 5.4 . 
Lemma 6.1. Let $k_{1} \geq 1$. There exists a map $S^{4 m-1} \stackrel{\bar{f}}{\longrightarrow} \Omega W$ such that the composite

$$
S^{4 m-1} \stackrel{\bar{f}}{\longrightarrow} \Omega W \stackrel{\Omega q}{\longrightarrow} \Omega V
$$

induces a map sending a generator $\iota_{2 m-1} \in H_{*}\left(S^{4 m-1} ; \mathbb{Z}_{p}\right)$ to $v^{2} \in H_{\star}\left(\Omega V ; \mathbb{Z}_{p}\right)$.

Proof. Let $S^{4 m-2} \stackrel{\alpha}{\longrightarrow} P^{2 m}\left(p^{r_{1}}\right)$ be the attaching map for the $(4 m-1)$-cell of $V$, and $S^{4 m-2} \stackrel{\xi}{\longrightarrow} \bar{W}$ the attaching map for the $(4 m-1)$-cell of $W$. Notice the map $W \stackrel{q}{\longrightarrow} V$ is the extension of a map $\bar{W} \stackrel{\bar{q}}{\longrightarrow} P^{2 m}\left(p^{r_{i}}\right)$ fitting in a diagram of cofibration sequences

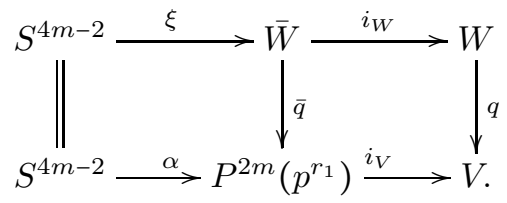

Proposition 5.3 implies $[\xi]$ has order $p^{r_{1}}$ in $\pi_{4 m-2}(\bar{W})$, since $r_{1}=\max \left\{r_{1}, \ldots, r_{k}\right\}$. Thus $\xi$ extends to a map $P^{4 m-1}\left(p^{r_{1}}\right) \stackrel{\bar{\xi}}{\longrightarrow} \bar{W}$.

Let $P^{4 m-2}\left(p^{r_{1}}\right) \stackrel{\bar{\xi}^{\prime}}{\longrightarrow} \Omega \bar{W}$ denote the adjoint of $\bar{\xi}$. Let $u^{\prime} \in H_{4 m-3}\left(P^{4 m-2}\left(p^{r_{1}}\right) ; \mathbb{Z}_{p}\right)$ and $v^{\prime} \in$ $H_{4 m-2}\left(P^{4 m-2}\left(p^{r_{1}}\right) ; \mathbb{Z}_{p}\right)$ be generators satisfying $\beta_{r}\left(v^{\prime}\right)=u^{\prime}$. By the above diagram of cofibrations, $\Omega \bar{q} \circ \bar{\xi}^{\prime}$ restricted to $S^{4 m-3}$ is the adjoint of $\alpha$, so Corollary 5.1 implies

$$
\left(\Omega \bar{q} \circ \bar{\xi}^{\prime}\right)_{*}\left(u^{\prime}\right)=[u, v]
$$

for some choice of our generator $u^{\prime}$.

When $m>2, H_{4 m-2}\left(\Omega P^{2 m}\left(p^{r_{1}}\right) ; \mathbb{Z}_{p}\right)$ is 1-dimensional vector space with a basis given by $v^{2}$. Thus $\left(\Omega \bar{q} \circ \bar{\xi}^{\prime}\right)_{*}\left(v^{\prime}\right)=k v^{2}$ for some $k$, and

$$
k[u, v]=\beta_{r_{1}}\left(k v^{2}\right)=\beta_{r_{1}}\left(\left(\Omega \bar{q} \circ \bar{\xi}^{\prime}\right)_{*}\left(v^{\prime}\right)\right)=\left(\Omega \bar{q} \circ \bar{\xi}^{\prime}\right)_{*}\left(\beta_{r_{1}}\left(v^{\prime}\right)\right)=\left(\Omega \bar{q} \circ \bar{\xi}^{\prime}\right)_{*}\left(u^{\prime}\right)=[u, v],
$$

so $k=1$. Therefore

Consider the composite

$$
\left(\Omega \bar{q} \circ \bar{\xi}^{\prime}\right)_{*}\left(v^{\prime}\right)=v^{2} .
$$

$$
f: P^{4 m-2}\left(p^{r_{1}}\right) \stackrel{\bar{\xi}^{\prime}}{\longrightarrow} \Omega \bar{W} \stackrel{\Omega i_{W}}{\longrightarrow} \Omega W .
$$

Now $\Omega q \circ f$ is homotopic to $\Omega i_{V} \circ \Omega \bar{q} \circ \bar{\xi}^{\prime}$, and since $H_{*}\left(\Omega V ; \mathbb{Z}_{p}\right) \cong T(u) \otimes T(v)$ such that $H$-map $\Omega P^{2 m}\left(p^{r_{1}}\right) \stackrel{\Omega i_{V}}{\longrightarrow} \Omega V$ induces a map on mod- $p$ homology modelled by the algebra map $T(u, v) \longrightarrow$ $T(u) \otimes T(v)$,

$$
(\Omega q \circ f)_{*}\left(v^{\prime}\right)=\left(\Omega i_{V}\right)_{*} \circ\left(\Omega \bar{q} \circ \bar{\xi}^{\prime}\right)_{*}\left(v^{\prime}\right)=\left(\Omega i_{V}\right)_{*}\left(v^{2}\right)=v^{2} .
$$

Notice $f$ factors through the quotient map $P^{4 m-2}\left(p^{r_{1}}\right) \longrightarrow S^{4 m-2}$, as the restriction of $f$ to the bottom sphere $S^{4 m-3}$ is null homotopic, since it is the adjoint of the (null homotopic) composite $S^{4 m-2} \stackrel{\xi}{\longrightarrow} \bar{W} \stackrel{i_{W}}{\longrightarrow} W$. Thus $f$ extends to a map $S^{4 m-2} \stackrel{\bar{f}}{\longrightarrow} \Omega W$ so that $\bar{f}_{*}\left(\iota_{4 m-2}\right)=f_{*}\left(v^{\prime}\right)$. Therefore

$$
(\Omega q \circ \bar{f})_{*}\left(\iota_{4 m-2}\right)=(\Omega q \circ f)_{*}\left(v^{\prime}\right)=v^{2} .
$$

This completes the proof.

Corollary 6.2. The map $\Omega W \stackrel{\Omega q}{\longrightarrow} \Omega V$ has a right homotopy inverse.

Proof. Assume $k_{1} \geq 1$ for now. By Theorem 3.5 there is a Hopf algebra isomorphism

$$
H_{*}\left(\Omega V ; \mathbb{Z}_{p}\right) \cong T(u) \otimes T(v),
$$

and the looped inclusion $\Omega P^{2 m}\left(p^{r_{1}}\right) \stackrel{\Omega i_{V}}{\longrightarrow} \Omega V$ induces a map on mod- $p$ homology modelled by the algebra map $T(u, v) \longrightarrow T(u) \otimes T(v)$ sending $u$ to $u$ and $v$ to $v$. Dependning on our construction of the map $W \stackrel{q}{\longrightarrow} V$ at the start of this section, we can take an inclusion $P^{2 m}\left(p^{r_{1}}\right) \stackrel{j}{\longrightarrow} W$ such that the composite $P^{2 m}\left(p^{r_{1}}\right) \stackrel{j}{\longrightarrow} W \stackrel{q}{\longrightarrow} V$ is homotopic to the inclusion $i_{V}$. Now consider the composite

$$
\phi: S^{2 m-1}\left\{p^{r_{1}}\right\} \stackrel{h}{\longrightarrow} \Omega P^{2 m}\left(p^{r_{1}}\right) \stackrel{\Omega j}{\longrightarrow} \Omega W .
$$


The map $h_{*}$ is modelled by taking $H_{*}\left(S^{2 m-1}\left\{p^{r_{1}}\right\} ; \mathbb{Z}_{p}\right)$ isomorphically onto the left $T(u)$-submodule of $T(u, v)$ with basis $\{1, v\}$, so $(\Omega q \circ \phi)_{*}$ is modelled by an isomorpism onto the left $T(u)$-submodule of $T(u) \otimes T(v)$ with basis $\{1, v\}$.

From Lemma 6.1 one has a map $S^{4 m-2} \stackrel{\bar{f}}{\longrightarrow} \Omega W$ satisfying $(\Omega q \circ \bar{f})_{*}\left(\iota_{4 m-2}\right)=v^{2} \in H_{*}\left(\Omega V ; \mathbb{Z}_{p}\right)$. Since $\Omega W$ is an $H$-space, by the universal property of the James construction for $\Omega S^{4 m-1} \bar{f}$ extends to an $H$-map

$$
\tilde{f}: \Omega S^{4 m-1} \longrightarrow \Omega W,
$$

and $(\Omega q \circ \tilde{f})_{*}$ induces an isomorphism onto the subalgebra of $H_{*}\left(\Omega V ; \mathbb{Z}_{p}\right)$ generated by $v^{2}$.

One now sees that the product

$$
S^{2 m-1}\left\{p^{r_{1}}\right\} \times \Omega S^{4 m-1} \stackrel{\phi \times \tilde{f}}{\longrightarrow} \Omega W \times \Omega W \stackrel{\Omega q \times \Omega q}{\longrightarrow} \Omega V \times \Omega V \stackrel{\text { mult. }}{\longrightarrow} \Omega V
$$

induces an isomorphism on mod- $p$ homology, and therefore is a homotopy equivalence. Since $\Omega q$ is an $H$-map, this homotopy equivalence is homotopic to the composite

$$
S^{2 m-1}\left\{p^{r_{1}}\right\} \times \Omega S^{4 m-1} \stackrel{\phi \times \tilde{f}}{\longrightarrow} \Omega W \times \Omega W \stackrel{\text { mult. }}{\longrightarrow} \Omega W \stackrel{\Omega q}{\longrightarrow} \Omega V
$$

and so $\Omega q$ has a right homotopy inverse.

Now consider $k_{1}=0$. Let $S^{2 m-1} \vee S^{2 m} \longrightarrow W$ be the inclusion inducing an isomorphism on mod- $p$ homology onto the subgroups generated by $x_{1}$ and $y_{1}$. Then the composite

$$
i^{\prime}: S^{2 m-1} \vee S^{2 m} \longrightarrow W \stackrel{q}{\longrightarrow} V
$$

is the inclusion of the $(4 m-2)$-skeleton of $V$. As we saw in the proof of Lemma 5.6, $\Omega i^{\prime}$ has a right homotopy inverse, and we are done.

It will be very covenient to make a change in basis in the proof of the next lemma. Depending on which of the four cases the matrix $A_{z^{*}}$ satisfies, as described at the start of this section, we change our basis $\mathbb{Z}_{p}\left\{x_{1}, x_{2}, x_{3}, \ldots x_{k}\right\}$ and $\mathbb{Z}_{p}\left\{y_{1}, y_{2}, y_{3}, \ldots y_{k}\right\}$ to $\mathbb{Z}_{p}\left\{a_{1}, a_{2}, a_{3}, \ldots a_{k}\right\}$ and $\mathbb{Z}_{p}\left\{b_{1}, b_{2}, b_{3}, \ldots b_{k}\right\}$ so that for $i \geq 2$ the following are satisfied:

$$
\begin{gathered}
q_{*}\left(a_{1}\right)=x, q_{*}\left(b_{1}\right)=y ; \beta_{r_{1}}\left(b_{1}\right)=a_{1} \text { if } 1 \leq k_{1} \\
q_{*}\left(a_{i}\right)=0, q_{*}\left(b_{i}\right)=0 ; \beta_{r_{i}}\left(b_{i}\right)=a_{i} \text { if } i \leq k_{1} ; \\
a_{1}^{*} b_{1}^{*}=c z^{*} \in H^{4 m-1}\left(W ; \mathbb{Z}_{p}\right) ;
\end{gathered}
$$

for some integer $c$ prime to $p$.

Since $q_{*}\left(x_{i}\right)=0$ and $q_{*}\left(y_{i}\right)=0$ when $i>2$, we can set $a_{i}=x_{i}$ and $b_{i}=y_{i}$. When the first case is satisfied, or when $k_{1}=0$, we may leave our previous basis as it was. For parts $(a),(b)$, and $(c)$ of the second case, by inspection we can set: $a_{1}=x_{2}, b_{1}=y_{2}, a_{2}=x_{1}$, and $b_{2}=y_{1} ; a_{1}=\frac{1}{2}\left(x_{1}+x_{2}\right)$, $b_{1}=\frac{1}{2}\left(y_{1}+y_{2}\right), a_{2}=x_{1}-x_{2}$, and $b_{2}=y_{1}-y_{2} ; a_{1}=x_{1}, b_{1}=y_{1}+y_{2}, a_{2}=x_{1}-x_{2}$, and $b_{2}=-y_{2}$, respectively.

Let $F$ be the homotopy fiber of $W \stackrel{q}{\longrightarrow} V$, and

$$
\Omega V \stackrel{\delta}{\longrightarrow} F \longrightarrow W
$$

be the induced principal homotopy fibration sequence, meaning there exists a left action

$$
\mu: \Omega V \times F \longrightarrow F
$$

such that the following diagram commutes up to homotopy

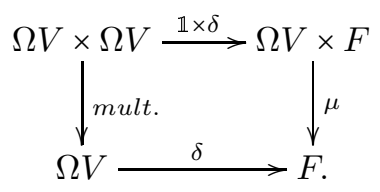


Lemma 6.3. There is isomorphism of left $H_{*}\left(\Omega V ; \mathbb{Z}_{p}\right)$-modules

$$
H_{*}\left(F ; \mathbb{Z}_{p}\right) \cong \mathbb{Z}_{p}\left\{a_{i}, b_{i} \mid 2 \leq i \leq k\right\} \otimes H_{*}\left(\Omega V ; \mathbb{Z}_{p}\right),
$$

where $\left|a_{i}\right|=2 m-1,\left|b_{i}\right|=2 m, \beta_{r_{i}}\left(b_{i}\right)=a_{i}$ when $i \leq k_{1}$, and the left action of $H_{*}\left(\Omega V ; \mathbb{Z}_{p}\right)$ is induced by $\mu$.

Proof. Recall the Hopf algebra isomorphism $H_{*}\left(\Omega V ; \mathbb{Z}_{p}\right) \cong T(u) \otimes T(v)$ from Theorem 3.5 . That is, $H_{*}\left(\Omega V ; \mathbb{Z}_{p}\right)$ is the polynomial algebra over $\mathbb{Z}_{p}$ generated by $u$ and $v$, where $u, v \in H_{*}\left(\Omega V ; \mathbb{Z}_{p}\right)$ are the transgressions of $x, y \in H_{*}\left(V ; \mathbb{Z}_{p}\right)$ in the mod- $p$ homology Serre spectral sequence for the path fibration of $V$.

The mod- $p$ homology spectral sequence $E$ for the principal homotopy fibration $\Omega V \stackrel{\delta}{\longrightarrow} F \longrightarrow W$ is a spectral sequence of left $H_{*}\left(\Omega V ; \mathbb{Z}_{p}\right)$-modules, with

$$
E_{*, *}^{2} \cong H_{*}\left(W ; \mathbb{Z}_{p}\right) \otimes H_{*}\left(\Omega V ; \mathbb{Z}_{p}\right),
$$

and left action induced by $\mu$. Differentials commute with the left action of $H_{*}\left(\Omega V ; \mathbb{Z}_{p}\right)$, that is $d^{n}(f \otimes g h)=(1 \otimes g) d^{n}(f \otimes h)$ whenever it makes sense.

Notice the generators $a_{i} \otimes 1, b_{i} \otimes 1 \in E_{*, 0}^{2}$ are transgressive since $\bar{W}$ is a suspension. Since $q_{*}\left(a_{i}\right)=$ $q_{*}\left(x_{i}\right)=0$ and $q_{*}\left(b_{i}\right)=q_{*}\left(y_{i}\right)=0$ for $i>2$, and likewise $q_{*}\left(a_{2}\right)=0$ and $q_{*}\left(b_{2}\right)=0$,

$$
d^{2 m-1}\left(a_{i} \otimes g\right)=0, d^{2 m}\left(b_{i} \otimes g\right)=0
$$

for every $g \in H_{*}\left(\Omega V ; \mathbb{Z}_{p}\right)$ and $i>1$. Since $q_{*}\left(a_{1}\right)=x$ and $q_{*}\left(b_{1}\right)=y$, and since $u, v \in H_{*}\left(\Omega V ; \mathbb{Z}_{p}\right)$ are the transgressions of $x, y \in H_{*}\left(V ; \mathbb{Z}_{p}\right)$,

$$
d^{2 m-1}\left(a_{1} \otimes 1\right)=1 \otimes u, d^{2 m}\left(b_{1} \otimes 1\right)=1 \otimes v .
$$

Notice $H_{*}\left(\Omega V ; \mathbb{Z}_{p}\right)$ is generated by elements of the form $v^{l}$ or $u g$ for monomials $g \in H_{*}\left(\Omega V ; \mathbb{Z}_{p}\right)$. Since

$$
d^{2 m-1}\left(a_{1} \otimes g\right)=(1 \otimes g) d^{2 m-1}\left(a_{1} \otimes 1\right)=(1 \otimes g)(1 \otimes u)=1 \otimes g u,
$$

no element $1 \otimes g u$ survives to $E_{0, *}^{2 m}$. Likewise, elements $1 \otimes v^{l}$ do not survive to $E_{0, *}^{2 m+1}$ since

$$
d^{2 m}\left(b_{1} \otimes v^{l-1}\right)=\left(1 \otimes v^{l-1}\right) d^{2 m}\left(b_{1} \otimes 1\right)=\left(1 \otimes v^{l-1}\right)(1 \otimes v)=1 \otimes v^{l} .
$$

So we see that no element in $E_{0, *}^{2}$ survives to $E_{0, *}^{\infty}$.

Now consider those elements of the form $b_{1} \otimes g u$. Since $1 \otimes g v u=1 \otimes g u v$ does not survive to $E_{0, *}^{2 m}$, we have

$$
d^{2 m}\left(b_{1} \otimes g u\right)=0 .
$$

Finally consider elements of the form $z \otimes g$. Note $d^{i}(z \otimes g)=0$ for $i<2 m-1$ for degree reasons. Let the integers $c_{1}, \ldots, c_{k}$ modulo $p$ be such that $b_{i}^{*} a_{1}^{*}=c_{i} z^{*}$. As mentioned before, $c_{1}=c$ is nonzero. Dualizing to the mod- $p$ cohomology spectral sequence associated with our homotopy fibration, for each $i$,

$$
d_{2 m-1}\left(b_{i}^{*} \otimes u^{*}\right)=d_{2 m-1}\left(b_{i}^{*} \otimes 1\right)\left(1 \otimes u^{*}\right)+(-1)^{\left|b_{i}\right|}\left(b_{i}^{*} \otimes 1\right) d_{2 m-1}\left(1 \otimes u^{*}\right)=\left(b_{i}^{*} \otimes 1\right)\left(a_{1}^{*} \otimes 1\right)=c_{i}\left(z^{*} \otimes 1\right) .
$$

Dualizing in the other direction, we have (denoted by $\zeta$ )

$$
\zeta=d^{2 m-1}(z \otimes 1)=\sum_{i=1}^{k} c_{i}\left(b_{i} \otimes u\right)
$$

so for each $g \in H_{*}\left(\Omega V ; \mathbb{Z}_{p}\right)$

$$
d^{2 m-1}(z \otimes g)=\sum_{i=1}^{k} c_{i}\left(b_{i} \otimes g u\right)=(1 \otimes g) \zeta .
$$

Therefore since $c_{1} \neq 0, b_{1} \otimes g u$ is identified with a linear combination of elements $b_{i} \otimes g u$ for $i>1$ in $E_{2 m, *}^{2 m}$.

All the differentials in this spectral sequence have now been computed. Summarizing all the above information, we see that no element in $E_{0, *}^{2}$ survives to $E_{0, *}^{\infty}$, elements of the form $a_{i} \otimes g$ for $i>1$ generate the kernel of $E_{2 m-1, *}^{2 m-1} \stackrel{d^{2 m-1}}{\longrightarrow} E_{0, *}^{2 m-1}$, and elements of the form $b_{i} \otimes g$ for $i>1$ and $b_{1} \otimes g u$ generate the kernel of $E_{2 m, *}^{2 m} \stackrel{d^{2 m}}{\longrightarrow} E_{0, *}^{2 m}$. But $b_{1} \otimes g u$ is identified with a linear combination of $b_{i} \otimes g u$ 
for $i>1$ in $E_{2 m, *}^{2 m}$, so $b_{i} \otimes g$ for $i>1$ generate this kernel. Since no nonzero linear combination of elements of the form $a_{i} \otimes g$ and $b_{i} \otimes g$ for $i>1$ is in the image of a differential, one sees $E_{*, *}^{\infty}$ is generated by a basis of elements $a_{i} \otimes g$ and $b_{i} \otimes g$ for $i>1$ and monomials $g \in H_{\star}\left(\Omega V ; \mathbb{Z}_{p}\right)$. The result follows.

Let

$$
J=\bigvee_{i=2}^{k_{1}} P^{2 m}\left(p^{r_{i}}\right) \vee \bigvee_{i=k_{1}+1}^{k}\left(S^{2 m-1} \vee S^{2 m}\right)
$$

This is the $(4 m-2)$-skeleton $\bar{W}$ of $W$ with $P^{2 m}\left(p^{r_{1}}\right)$ quotiented out. Our previous work amounts to the following:

\section{Theorem 6.4.}

(i) If $k_{1} \geq 1$ there is a homotopy equivalence

$$
\Omega W \simeq S^{2 m-1}\left\{p^{r_{1}}\right\} \times \Omega S^{4 m-1} \times \Omega\left(J \vee\left(J \wedge\left(S^{2 m-1}\left\{p^{r_{1}}\right\} \times \Omega S^{4 m-1}\right)\right)\right),
$$

where the right-hand space is taken to be a weak product.

(ii) Similarly, if $k_{1}=0$ there is a homotopy equivalence

$$
\Omega W \simeq \Omega S^{2 m-1} \times \Omega S^{2 m} \times \Omega\left(J \vee\left(J \wedge\left(\Omega S^{2 m-1} \times \Omega S^{2 m}\right)\right)\right) .
$$

Proof of part $(i)$. By Corollary $6.2 \Omega W \stackrel{\Omega q}{\longrightarrow} \Omega V$ has a right homotopy inverse, so the homotopy fibration sequence $\Omega F \longrightarrow \Omega W \stackrel{\Omega q}{\longrightarrow} \Omega V$ is split. Therefore

$$
\Omega W \simeq \Omega V \times \Omega F \simeq S^{2 m-1}\left\{p^{r_{1}}\right\} \times \Omega S^{4 m-1} \times \Omega F,
$$

where $\Omega V \simeq S^{2 m-1}\left\{p^{r_{1}}\right\} \times \Omega S^{4 m-1}$ by Theorem 5.5.

Let $\Omega V \stackrel{s}{\longrightarrow} \Omega W$ be a right homotopy inverse of $\Omega q$, and $\Omega V \stackrel{\delta}{\longrightarrow} F$ be the connecting map in the homotopy fibration sequence (17). Since $\delta \circ \Omega q$ is null homotopic, we have $\delta \simeq \delta \circ \Omega q \circ s \simeq *$, so $\delta$ is null homotopic as well. Now by Lemma 6.3 the $2 m$-skeleton of $F$ is the wedge sum $J$ in equation (19).

Define the composite

$$
\lambda: \Omega V \times J \stackrel{\mathbb{1} \times j}{\longrightarrow} \Omega V \times F \stackrel{\mu}{\longrightarrow} F,
$$

where $j$ is the inclusion of the $2 m$-skeleton. Notice the composite

$$
\Omega V \times * \stackrel{\mathbb{1} \times *}{\longrightarrow} \Omega V \times J \stackrel{\lambda}{\longrightarrow} F
$$

is null homotopic, as it is homotopic to $\delta \simeq *$ by diagram (18). Therefore one obtains an extension $\bar{\lambda}$ of $\lambda$ in the following homotopy commutative diagram

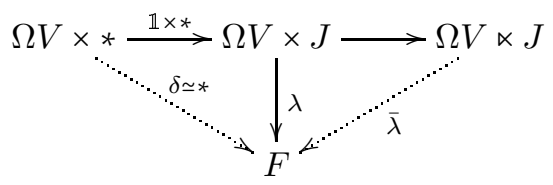

where the half-smash product $\Omega V \ltimes J$ is by definiton the cofiber of the inclusion $\Omega V \times * \subset \Omega V \times J$. By Lemma 6.3

$$
H_{*}\left(F ; \mathbb{Z}_{p}\right) \cong \bar{H}_{*}\left(J ; \mathbb{Z}_{p}\right) \otimes H_{*}\left(\Omega V ; \mathbb{Z}_{p}\right) \cong H_{*}\left(\Omega V \ltimes J ; \mathbb{Z}_{p}\right) .
$$

Observe that $\lambda$ restricts to an isomorphism of the submodule

$$
\bar{H}_{*}\left(J ; \mathbb{Z}_{p}\right) \otimes H_{*}\left(\Omega V ; \mathbb{Z}_{p}\right) \subseteq H_{*}\left(\Omega V \times J ; \mathbb{Z}_{p}\right)
$$

onto $H_{*}\left(F ; \mathbb{Z}_{p}\right)$, so $\bar{\lambda}$ induces an isomorphism on mod-p homology. Therefore

$$
F \simeq \Omega V \ltimes J
$$

Now applying the well known general splitting of half-smash products

$$
B \ltimes(\Sigma A)=(\Sigma A) \rtimes B \simeq(\Sigma A) \vee(\Sigma A \wedge B),
$$


one obtains

$$
\begin{aligned}
F & \simeq \Omega V \ltimes J \\
& \simeq J \vee(J \wedge \Omega V) \\
& \simeq J \vee\left(J \wedge\left(S^{2 m-1}\left\{p^{r}\right\} \times \Omega S^{4 m-1}\right)\right),
\end{aligned}
$$

and we are done

Proof of part (ii). The proof is identical to that of part $(i)$, except with Theorem 5.6 used in place of Theorem 5.5

Proof of Theorem 2.3 and Theorem 1.1 part $(i)$. For each of the homotopy equivalences in Theorem 6.4 the homotopy type of the right-hand weak product is uniquely determined by the integers $k$ and $k_{1}$, and the integers $r_{1}, \ldots, r_{k_{1}}$. The ordering is arbitrary, but we selected it so that $r_{1}=\max \left\{r_{1}, \ldots, r_{k_{1}}\right\}$ when $k_{1}>0$. As is clearly seen in Equation (2), these integers correspond uniquely to the homotopy type of $\bar{W}$. Therefore the homotopy types of the right-hand weak products in Theorem 6.4 are uniquely determined by the homotopy type of $\bar{W}$.

It is clear that any two $[W],\left[W^{\prime}\right] \in \mathcal{T}_{k, 2 m}^{p}$ satisfy conditions (1) and (2) in Theorem 1.1 if and only if $\bar{W} \simeq \bar{W}^{\prime}$. The result follows by application of Theorem 6.4 .

\section{The Integral Case}

In this section spaces are not assumed to be localized. When we say that the localization of map or space at a prime $p$ is another map or space, we will mean that they are the same at least up to homotopy equivalence.

Let $\mathcal{P}=\left\{p_{1}, p_{2}, \ldots\right\}$ be the set of all prime numbers, and $M$ be a manifold as in part $(i i)$ of Theorem 1.1. The uniqueness up to homotopy type of Moore spaces implies the $(4 m-2)$-skeleton $\bar{M}$ of $M$ splits as a finite wedge of Moore spaces

$$
\bar{M} \simeq \bigvee_{i}\left(\bigvee_{j} P^{2 m}\left(q_{i}^{r_{i, j}}\right)\right),
$$

where $\mathcal{Q}=\left\{q_{1}, q_{2}, \ldots\right\} \subset\left\{p_{1}, p_{2}, \ldots\right\}$ is some subset of odd prime numbers. We may as well assume the homotopy type of $M$ (which might now longer be a manifold) has been selected so the $\bar{M}$ is homeomorphic to the above wedge of Moore spaces.

When localized at some $p, \bmod -q$ Moore spaces are contractible whenever $q$ prime to $p$. Then $\bar{M}_{\left(q_{i}\right)}$ is homotopy equivalent to a wedge of the mod $-q_{i}$ Moore spaces in the above splitting. On the other hand, $\bar{M}_{(p)}$ is contractible when localized at any $p \in \mathcal{P}-\mathcal{Q}$, which implies $M_{(p)} \simeq S_{(p)}^{4 m-1}$.

We will need to lift some of the $p$-local maps constructed in the previous section to ones that are integral. This is perhaps best done by following through the same constructions, all the while keeping in mind we are no longer localized. First, using the recipe for the construction of the map $W \stackrel{q}{\longrightarrow} V$ in (15), we can collapse or fold the Moore spaces in the $(4 m-2)$-skeleton $\bar{M}$ to obtain a map

$$
\rho: M \longrightarrow N
$$

whose localization at each prime $p \in \mathcal{Q}$ is the map $q$, and whose localization at each $p \in \mathcal{P}-\mathcal{Q}$ is a homotopy equivalence $M \simeq S_{(p)}^{4 m-1} \stackrel{\simeq}{\longrightarrow} S_{(p)}^{4 m-1} \simeq N$. Then the $(4 m-2)$-skeleton of $N$ is a finite wedge

$$
\bar{N} \simeq \bigvee_{i} P^{2 m}\left(q_{i}^{s_{i}}\right)
$$

where $s_{i}=\max _{j}\left\{r_{i, j}\right\}$. 
Let $Q=\prod_{i} S^{2 m-1}\left\{q_{i}^{s_{i}}\right\}$ and $Q^{\prime}=\prod_{i} \Omega P^{2 m}\left(q_{i}^{s_{i}}\right)$. The $p$-local map $h$ in Section 5 can be lifted to an integral map $h^{\prime}$ that is a choice of lift in the digram of homotopy fibrations sequence

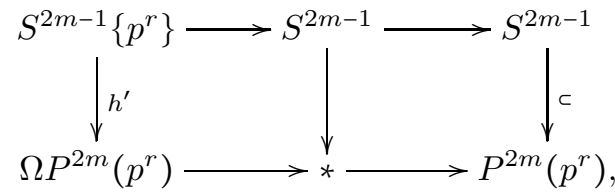

for this is how $h$ was constructed in [4. Taking products of these maps defines the obvious map $\Omega Q \longrightarrow \Omega Q^{\prime}$. Using the Hilton-Milnor homotopy decomposition of $\Omega \bar{N}$ gives a map $Q^{\prime} \longrightarrow \Omega \bar{N}$ that is a left homotopy inverse of the canonical looped inclusion $\Omega \bar{N} \longrightarrow Q^{\prime}$. Now we define the composite

$$
\eta: Q \longrightarrow Q^{\prime} \longrightarrow \Omega \bar{N} \longrightarrow \Omega M
$$

where the last map is the looped inclusion.

Next, construct a map

$$
\tilde{g}: \Omega S^{4 m-1} \longrightarrow \Omega M,
$$

which is the integral analogue of the map $\tilde{f}$ in the proof of Corollary 6.2. The construction begins along the same lines as that of the map $\bar{f}$ in Lemma 6.1. First take the attaching map $S^{4 m-2} \stackrel{\xi}{\longrightarrow} \bar{M}$

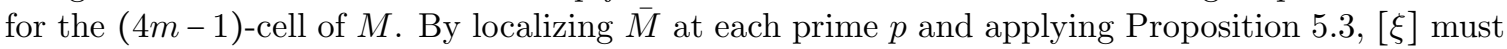
be of order $s=\prod_{i} q_{i}{ }^{s_{i}}$, so $\xi$ factors through a map $P^{4 m-1}(s) \stackrel{\bar{\xi}}{\longrightarrow} \bar{M}$. We then let $P^{4 m-2}(s) \stackrel{\bar{\xi}^{\prime}}{\longrightarrow} \Omega \bar{M}$ be the adjoint of $\bar{\xi}$. The restriction of the map

$$
g: P^{4 m-2}(s) \stackrel{\bar{\xi}^{\prime}}{\longrightarrow} \Omega \bar{M} \longrightarrow \Omega M
$$

to the bottom sphere $S^{4 m-3}$ is null homotopic since it is the adjoint of $S^{4 m-2} \stackrel{\xi}{\longrightarrow} \bar{M} \longrightarrow M$, which itself is null homotopic. Then $g$ factors through a map

$$
\bar{g}: S^{4 m-2} \longrightarrow \Omega M,
$$

By the universal property of the James construction for $\Omega S^{4 m-1}, \bar{g}$ extends to the map $\tilde{g}$ detailed above.

The following is well known (see [13] for example):

Proposition 7.1. A map $X \longrightarrow Y$ of finite type 1-connected $C W$-complexes is a homotopy equivalence if and only if it induces a homotopy equivalence localized at each prime $p$.

Using this, and our preceeding work localized at odd primes $p$, we prove the integral classification in part $(i i)$ of Theorem 1.1 .

Proof of Theorem 1.1 part (ii). Combine our maps to obtain the composite

$$
\psi: Q \times \Omega S^{4 m-1} \stackrel{\eta \times \tilde{g}}{\longrightarrow} \Omega M \times \Omega M \stackrel{\text { mult. }}{\longrightarrow} \Omega M \stackrel{\Omega \rho}{\longrightarrow} \Omega N .
$$

Notice a $S^{2 m}\left\{q^{r}\right\}$ factor in $Q$ has trivial mod- $p$ homology when $q$ is prime to $p$, and thus is contractible when localized at $p$. The maps $\eta$ and $\tilde{g}$ localize at $p \in \mathcal{Q}$ to the corresponding maps $\phi$ and $\tilde{f}$ in composite the composite (16), so $\psi_{(p)}$ is the composite (16). On the other hand, $\psi_{(p)}$ reduces to a homotopy equivalence $\Omega S_{(p)}^{4 m-1} \stackrel{\simeq}{\longrightarrow} \Omega N_{(p)}$ when localized at $p \in \mathcal{P}-\mathcal{Q}$. Now applying Lemma 7.1 $\psi$ is itself a homotopy equivalence.

Let $G$ be the homotopy fiber of $M \stackrel{\rho}{\longrightarrow} N$. The homotopy fibration sequence

$$
\Omega G \longrightarrow \Omega M \stackrel{\Omega \rho}{\longrightarrow} \Omega N
$$

therefore has a homotopy cross-section given by the homotopy equivalence $\psi$, and as such there are homotopy equivalences

$$
\Omega G \times Q \times \Omega S^{4 m-1} \simeq \Omega G \times \Omega N \simeq \Omega M .
$$


The homotopy type of $Q$ clearly depends only on the homotopy type of the $(4 m-2)$-skeleton $\bar{M}$. To complete the proof, we need to show that the homotopy type of $\Omega G$ also depends only on that of $\bar{M}$.

Let $I$ be the quotient $\bar{M} / \bar{N}$. Localized at $p \in \mathcal{Q}, I_{(p)}$ is homotopy equivalent to the wedge $J$ in Theorem 6.4. The localization $G_{(p)}$ is contractible when $p \in \mathcal{P}-\mathcal{Q}$, since $\rho$ is a homotopy equivalence in this case. When $p \in \mathcal{Q}, G_{(p)}$ is the homotopy fiber $F$ in Theorem 6.4 and we have shown that the $2 m$-skeleton of $F$ is $J \simeq I_{(p)}$. Thus $I$ is the $2 m$-skeleton of $G$. We can now carry forward with a construction similar to the one in the proof of Theorem 6.4 and produce a map

$\Omega N \ltimes I \stackrel{\bar{\gamma}}{\longrightarrow} G$ whose localization $\bar{\gamma}_{(p)}$ at $p \in \mathcal{Q}$ is the homotopy equivalence $\Omega V \ltimes J \stackrel{\bar{\lambda}}{\longrightarrow} F$ in the proof thereof. Localized at primes $p \in \mathcal{Q}, \bar{\gamma}_{(p)}$ is also a homotopy equivalence, since here both $G_{(p)}$ and $(\Omega N \ltimes I)_{(p)} \simeq I_{(p)} \vee I_{(p)} \wedge \Omega N_{(p)}$ are contractible. Lemma 7.1 now implies

$$
G \simeq \Omega V \ltimes I .
$$

The homotopy type of $\bar{N}$ is depends only on the homotopy type of $\bar{M}$, and so the same applies for $I$, and consequently for $G$ and $\Omega G$ as well.

\section{REFERENCES}

1. D.J. Anick, Homotopy exponents for spaces of category two, Algebraic topology (Arcata, CA, 1986), Lecture Notes in Math., vol. 1370, Springer, Berlin, 1989, pp. 24-52. MR 1000365 (90c:55010)

2. M.G. Barratt, Spaces of finite characteristic, Quart. J. Math. Oxford Ser. (2) 11 (1960), 124-136. MR 0120647 (22 \#11396)

3. P. Bubenik, Free and semi-inert cell attachments, Trans. Amer. Math. Soc. 357 (2005), no. 11, 4533-4553. MR 2156720 (2006c:55007)

4. F.R. Cohen, J.C. Moore, and J.A. Neisendorfer, The double suspension and exponents of the homotopy groups of spheres, Ann. of Math. (2) 110 (1979), no. 3, 549-565. MR 554384 (81c:55021)

5. Y. Félix and J.C. Thomas, Effet d'un attachement cellulaire dans l'homologie de l'espace des lacets, Ann. Inst. Fourier (Grenoble) 39 (1989), no. 1, 207-224. MR 1011984 (90j:55012)

6. J. Grbić and J. Wu, Applications of combinatorial groups to Hopf invariant and the exponent problem, Algebr. Geom. Topol. 6 (2006), 2229-2255 (electronic). MR 2263065 (2007k:55010)

7. S. Halperin and J.-M. Lemaire, Suites inertes dans les algèbres de Lie graduées ("Autopsie d'un meurtre. II"), Math. Scand. 61 (1987), no. 1, 39-67. MR 929396 (89e:55022)

8. ㄴ The fibre of a cell attachment, Proc. Edinburgh Math. Soc. (2) 38 (1995), no. 2, 295-311. MR 1335875 (97c:55013)

9. K. Hess and J.-M. Lemaire, Nice and lazy cell attachments, J. Pure Appl. Algebra 112 (1996), no. 1, 29-39. MR 1402394 (97e:55006)

10. J.R. Klein, Poincaré duality spaces, Surveys on surgery theory, Vol. 1, Ann. of Math. Stud., vol. 145, Princeton Univ. Press, Princeton, NJ, 2000, pp. 135-165. MR 1747534 (2001b:57053)

11. J.-M. Lemaire, "Autopsie d'un meurtre” dans l'homologie d'une algèbre de châ̂nes, Ann. Sci. École Norm. Sup. (4) 11 (1978), no. 1, 93-100. MR 0500930 (58 \#18423)

12. J. Milnor, On simply connected 4-manifolds, Symposium internacional de topología algebraica International symposi um on algebraic topology, Universidad Nacional Autónoma de México and UNESCO, Mexico City, 1958, pp. 122-128. MR 0103472 (21 \#2240)

13. M. Mimura, G. Nishida, and H. Toda, Localization of CW-complexes and its applications, J. Math. Soc. Japan 23 (1971), 593-624. MR 0295347 (45 \#4413)

14. S. Sasao and H. Takahashi, Highly connected Poincaré complexes, Kodai Math. J. 2 (1979), no. 2, $139-147$. MR 548390 (80k:57035)

15. R. Stöcker, On the structure of 5-dimensional Poincaré duality spaces, Comment. Math. Helv. 57 (1982), no. 3, 481-510. MR 689075 (85b:57022)

16. C.T.C. Wall, Classification of (n-1)-connected 2n-manifolds, Ann. of Math. (2) 75 (1962), 163-189. MR 0145540 (26 \#3071)

School of Mathematics, University of Southampton,Southampton SO17 1BJ, United Kingdom

E-mail address: P.D.Beben@soton.ac.uk

Department of Mathematics, National University of Singapore, Block S17 (SOC1), 10, Lower Kent Ridge Road, SINGAPORE 119076

E-mail address: matwuj@nus.edu.sg 